\title{
Fractional Excitations in the Luttinger Liquid.
}

\author{
K.-V. Pham, M. Gabay and P. Lederer \\ Laboratoire de Physique des Solides, associé au CNRS \\ Université Paris-Sud \\ 91405 Orsay, France \\ (September 18, 2018)
}

\begin{abstract}
We reconsider the spectrum of the Luttinger liquid (LL) usually understood in terms of phonons (density fluctuations), and within the context of bosonization we give an alternative representation in terms of fractional states. This allows to make contact with Bethe Ansatz which predicts similar fractional states. As an example we study the spinon operator in the absence of spin rotational invariance and derive it from first principles: we find that it is not a semion in general; a trial Jastrow wavefunction is also given for that spinon state. Our construction of the new spectroscopy based on fractional states leads to several new physical insights: in the low-energy limit, we find that the $S_{z}=0$ continuum of gapless spin chains is due to pairs of fractional quasiparticle-quasihole states which are the 1D counterpart of the Laughlin FQHE quasiparticles. The holon operator for the Luttinger liquid with spin is also derived. In the presence of a magnetic field, spin-charge separation is not realized any longer in a LL: the holon and the spinon are then replaced by new fractional states which we are able to describe.
\end{abstract}

PACS Numbers: 71.10 Pm , 71.27+a

\section{INTRODUCTION.}

\section{A. Motivations of this work.}

One of the most striking property of some strongly correlated systems is fractionalization, that is the existence of elementary excitations carrying only part of the quantum numbers of the constituent particles of the system. The most famous example is probably the charge one-third Laughlin quasiparticle, which is the elementary excitation of the fractional quantum Hall fluid at filling $\nu=1 / 3$. Its existence was recently confirmed in a beautiful set of shot noise experiments 2 . The earliest example of fractionalization in condensed matter physics is however found in one dimension: the exact solution of the Hubbard model 3 by Bethe Ansat 1 revealed that the charge and spin of the electron split into two excitations with independent dynamics, known as the holon and the spinon. Faddeev and Takhtajan later showed that the same spinon is also the elementary excitation of the 1D Heisenberg model: the magnon (the usual Goldstone boson) is replaced by two spipgns generating a continuum for $\Delta S=1$ excitations 6 - This property of the Hubbard model is known as spin-charge separation and is generic of so-called Luttinger liquids (LL): LL constitute a universality class for gapless one dimensional models such as the Heisenberg chain, the Hubbard and $t-J$ models $\$$. Luttinger liquids are nonFermi liquids: Landau quasiparticles 9 are not elementary excitations of the LL and as a consequence the electron Green's function shows no quasiparticle pole (this property is true both for the LL with spin and for the spinless LL). Haldane, who coined the name of LL, conjectured that 1D gapless models would have the same low-energy physics as that of the Tomonaga-Luttinger model. For energies smaller than the bandwidth 10 12, the latter medel is a fixed point of the renormalization group (RG)13. In 1D, bosonization allows to transform the Tomonaga-Luttinger model into gaussian acoustic hamiltonian describing free phonon 14 ; the considerable success and popularity of bosonization stems from the fact that all the computations are straightforward because the effective hamiltonian is that of a free bosonic field. Another perspective on the LL is provided by conformal field theory (CFT) which describes two dimensional (or $1+1$ ) critical theories with conformal invariance; this has allowed to identify the Luttinger liquid universality class as the set of $c=1$ CFTs 15 , i.e. the set of all models which flow under RG towards the gaussian free boson hamiltonian 16. CFT has allowed to formalize the finite-size analysis of Luttinger liquids first introduced by Haldane 17 18 8 . In terms of the gaussian hamiltonian, the LL theory can be described as a phenomenological theory characterized by the following parameters: $u$ which is a velocity for collective modes and $K$ which is proportional to the compressibility of the system.

Yet, although the LL description is supposedly quite well established through the formalisms of bosonization or CFT, and despite the fact that exact solutions (Bethe Ansatz) show the existence of fractional states in the spectrum of several Luttinger liquids, there exists no systematic study of fractional excitations in the LL to the best of the authors' knowledge. What's more, in the framework of the bosonization formalism, it is sometimes stated that the only physically relevant excitations of a LL are phonons, since the effective hamiltonian is just that of acoustic phonons. As we show below this statement is incorrect. Conformal field theory is an alternative to bosonization which does stress the spectroscopic aspects: yet, application to the study of fractional excitations in a LL has been limited to the spinon in the case of $S U(2)$ symmetry, which is the situation relevant 
for the Heisenberg chain 19. Fractional excitations must exist in a Luttinger liquid if Bethe Ansatz is correct but as far as the authors are aware, the characterization of these very unconventional fractional states, through either bosonization or CFT, is mostly terra incognita as the following list of issues may show:

1) $\Delta S=1$ excitations for the Heisenberg chain form a continuum of pairs of spinons. When an Ising anisotropy is introduced, in the massless regime (with obvious notations: $\left.\left|J_{z}\right| \leq J_{x}\left(=J_{y}\right)\right)$ the continuum still exists and evolves smoothly as a function of the anisotropy $\Delta=J_{z} J_{x}$. The continuum is again ascribed to pairs of spinons2 20 . It is intuitively clear that these spinons should be in some sense deformations of the $S U(2)$ spinon. We will derive in this paper creation operators for these non$S U(2)$ spinons using the bosonization method.

In the case of $S U(2)$ symmetry, trial wavefunctions for these spinons can be found by making use of the exact solvability of the Haldane-Shastry (HS) chain21. The HS model shares the properties of the Heisenberg chain: it is a gapless $S U(2)$ symmetric spin chain with a continuum of spinop-excitations. Its ground state and spinon wavefunction 22 are remarkably similar to those one would write for bosonic Laughlin states at filling $\nu=1 / 2$ :

$$
\begin{aligned}
\Psi_{g s}\left(x_{1}, . ., x_{N}\right) & =\prod_{i<j}\left(z_{i}-z_{j}\right)^{2}, \\
\Psi_{\text {spinon }}\left(z_{0}\right) & =\prod_{i}\left(z_{i}-z_{0}\right) \prod_{i<j}\left(z_{i}-z_{j}\right)^{2}, \\
z_{i} & =\exp i \frac{2 \pi}{L} x_{i},
\end{aligned}
$$

where $x_{i}$ is the coordinate a spin down, and $x_{0}$ that of the spinon 23. We will exhibit a similar wavefunctions for the spinon in the absence of $S U(2)$ symmetry. $S U(2)$ spinons are semions 22 (anyons with a statistics intermediate between that of fermions and bosons): we will show that the statistics is affected when an anisotropy is introduced.

A continuym is also found by Bethe Ansatz for $\Delta S_{z}=$ 0 transitions 24. Low-lying excitations are described in that approach as two-strings states in the string formalism customary to Bethe Ansatz. This description is similar to that given for the $\Delta S=1$ continuum of the isotropic chain. In the latter case it is quite clear that a spin one-half should be ascribed to each of the (pseudo) "hole" states in the string, which leads to the spinon interpretation since each state should contribute symmetrically to the spin-flip 6 . For $\Delta S_{z}=0$ transitions, the total $z$ spin components of the excitations add up to 0 and the continuum results from the excitation of particle-hole pairs. For the isotropic chain, this continuum is generated by spin $1 / 2$ spinon-antispinon pairs. By contrast, for the $X Y$ chain the $\Delta S_{z}=0$ continuum is due to particle-hole pairs of magnon-like spin $S_{z}=1$ excitations. The case of the isotropic Heisenberg chain for which spin $1 / 2$ spinons are involved both in the $\Delta S_{z}=0$ and the $\Delta S_{z}=1$ continuum, is therefore incidental. The important lesson to be learned is that in the presence of an Ising anisotropy, the $\Delta S_{z}=1$ and $\Delta S_{z}=0$ continua may involve differentfractional spin states: in the first case we have spinons 20 , but in the second case the spinon identification is not always correct. What happens in the case of an arbitrary anisotropy will be dealt with in this paper.

2) The holon appearing in the exact solution of the Hubbard model is a spinless charge one excitation. The issues raised for the spinon (operator, wavefunction, statistics) extend naturally to the holon.

3) Spin-charge separation is an asymptotic property of the Hubbard model valid in the low-energy limit. When a magnetic field is applied Frahm and Korepin found that spin-charge jecoupling was not realized even in the lowenergy limit25. In this paper we derive the new excitations replacing the holon and the spinon.

4) the context of the Calogero-Sutherland (CS) mode 26 the existence of fractional excitations similar to Laughlin quasiparticles was suggested27. In a variant of the standard LL known as the chiral LL used to describe edges of a FQHE sample, Laughlin quasiparticles do appear but the existence of such states follows from that of the same excitations in the bulk 28 . In the CS model the proposal was triggered by the similarity of the ground state with that of the 2D Laughlin wavefunctions and by special selection rules. The ground state is 26 :

$$
\begin{aligned}
\Psi\left(x_{1}, . ., x_{N}\right) & =\prod_{i<j}\left|z_{i}-z_{j}\right|^{\lambda}, \\
z_{i} & =\exp i \frac{2 \pi}{L} x_{i}
\end{aligned}
$$

where $\lambda$ is a coupling constant for the $1 / r^{2}$ interaction potential of the CS model. The CS model is a LL18 and the LL parameter is just $K=1 / \lambda$. A pseudo-particle formalism similar to that of Bethe Ansatz can be introduced and for the restricted case of rational couplings $\lambda=p / q$ special selection rules are found for the dynamical structure factor: $p$ pseudo-holes (a pseudo-hole is a hole in the Fermi sea of pseudo-momenta) must be accompanied in any excitation by $q$ pseudo-particles 29 . For a charge $(-1)$ pseudo-particle, this means one has a charge $1 / \lambda=K$ for the pseudo-hole. In the interpretation of those selection rules it is proposed to view the CS model as a gas of non-interacting pseudo-particles with anyonic statistics $\pi \lambda$ and one rewrites the ground state as an anyonic wavefunction $\prod_{i<j}\left(z_{i}-z_{j}\right)^{\lambda}$. The pseudo-holes are particle-hole conjugate of these anyons: the main modification with the non-interacting case being the new selection rule; a wavefunction for these pseudo-holes consistent with those interpretations is then $\prod_{i}\left(z_{i}-z\right) \prod_{i<j}\left(z_{i}-z_{j}\right)^{\lambda}$ which has the correct charge and statistics. The pseudo-hole is therefore identified as a Laughlin quasiparticle. It exists for rational couplings and carries the rational charge $1 / \lambda$. 
There are however several limitations to those views. Firstly, these considerations are only valid for rational couplings (the pseudo-particle selection rules can not be extended to irrational $\lambda$ ): the physics of the CS model is by contrast completely continuous with the coupling and does not discriminate between rational and irrational couplings. The impossibility to describe rational and irrational couplings on the same footing means the representation is not adequate. Secondly a disymmetry between particle and hole excitations is introduced.

In a parallel strand of ideas, Laughlin quasiparticles were also proposed in studies of transport in a LL. The basis of the argument is that for a LL with an impurity potential a charge $K$ and not a charge unity is backscattered at the impurity location (where $K$ is the LL parameter, i.e. the conductance of the LL) 30 . The impurity potential can be rewritten as a hopping potential for a charge $K$ state whose exchange statistics is $\pi K$ as seen from the commutation relations (i.e. anyonic). But for $K=1 /(2 n+1)$ these states are just those given by Wen for the Laughlin quasiparticles of his chiral LL: this suggests to identify these states as Laughlin quasiparticles. The main difficulty in that argument is that it relies on the introduction of an impurity potential in the LL: this obscures the question of the existence or not of a Laughlin quasiparticle in the pure non-chiral Luttinger liquid. In summary what is missing is a proof that states similar to Laughlin quasiparticles might be exact eigenstates of the LL boson hamiltonian (i.e. of the RG fixed point in the low-energy limit). The existence of Laughlin quasiparticles for the non-chiral LL for arbitrary couplings must then be considered at this point as an unproved conjecture.

The long list of issues we have brought up in points $(1-4)$ above should convince the reader that a thorough discussion of fractional excitations for Luttinger liquids within the formalisms of bosonization or CFT remains to be done. This is what motivated us to re-examine in that paper the spectrum of Luttinger liquids. We want to stress that although the previous examples concern integrable models, the detailed physics of such integrable models is not really our main interest: what matters for us is the universal low-energy content of these theories and of course we will be unable to tell anything through bosonization on the high-energy physics. Although it seems to be taken for granted that the excitations of a LL are the holon and the spinon on account of Bethe Ansatz studies of the Hubbard model, we are not aware of any existence proof of such fractional excitations whenever the model is non-integrable: this is so because any proof must resort to the universality hypothesis, that is to the LL and bosonization frameworks. The theoretical formalism we wish to introduce aims at bridging that gap by focusing on the universal structure of fractional excitations of Luttinger liquids through the bosonization method.

The structure of the paper will be as follows: section I is an introduction to the topics considered in the pa- per. In subsection IB we give a short review of the LL physics in order to set the notations used throughout the paper; the issues discussed in the present subsection I A will be amplified in subsection IB 2 in which we present the standard view on excitations of the LL. In the next section II we will show that an alternative eigenstate basis can be built: that quasiparticle basis allows a natural discussion of fractional states. In section III, we will generalize our analysis to the LL with spin. When a magnetic field is added on to the Hubbard model, spin-charge separation no longer occurs 25 . The standard spin-charge separated Luttinger liquid theory is not applicable any more. We will introduce in subsection IIIB a general framework related to the $K$ topological matrix of Wen's chiral Luttinger liquid\$2 28 , which yields simple criteria of spin-charge separation in terms of a $Z_{2}$ symmetry: we will be able then in subsection III to derive the fractional excitations which replace the holon and the spinon. The general LL theory we have introduced will then be applied to the Hubbard model in a magnetic field in subsection IIID in which we explain the relation between our approach and the formalism of the dressed charge matrix due to Frahm and Korepin. Let us mention that section II of this paper expands on a short version which contained results in the case of a spinless LL32, whereas part III presents totally new material.

\section{B. The Luttinger liquid.}

\section{Notations.}

This section will define the notations employed throughout the paper. We exclusively deal with Luttinger liquids and therefore when considering some specific models such as the Heisenberg spin chain or the Hubbard model we implicitly assume that we are working in the LL part of their phase diagrams. The whole physics of the LL is embodied in the following hamiltonian:

$$
H_{B}=\frac{u}{2} \int_{0}^{L} d x K^{-1}(\nabla \Phi(x))^{2}+K(\nabla \Theta(x))^{2}
$$

supplemented by the so-called bosonization formulas. We work on a ring of length $L . u$ and $K$ are the LL parameters. $\Phi$ can be interpreted as a displacement field for phonons, while $\Theta$ is a superfluid phase; indeed the particle and current densities are defined as:

$$
\begin{aligned}
\rho(x)-\rho_{0} & =-\frac{1}{\sqrt{\pi}} \nabla \Phi(x), \\
j(x) & =\frac{1}{\sqrt{\pi}} \nabla \Theta(x) .
\end{aligned}
$$

Renormalized current: Actually $j(x)$ is a bare current density which corresponds to the correct one only in the non-interacting case $K=1$ : the continuity equation 
shows that the correct current density is renormalized and is

$$
j_{R}(x)=u K j(x)
$$

(the Fermi velocity has been set to unity). We will discuss in section II the meaning of such a renormalization.

The particle operators for bosons and right and left moving fermions respectively are given as:

$$
\begin{aligned}
\Psi_{B}(x) & =: \exp i \sqrt{\pi} \Theta(x): \\
\Psi_{F, R}(x) & =: \exp i \sqrt{\pi}(\Theta(x)-\Phi(x)): \exp i k_{F} x, \\
\Psi_{F, L}(x) & =: \exp i \sqrt{\pi}(\Theta(x)+\Phi(x)): \exp -i k_{F} x
\end{aligned}
$$

(in the following we will assume that these operators are normal ordered). $k_{F}=\pi N_{0} / L$ is the Fermi momentum where $N_{0}$ is the number of particles which is fixed by the chemical potential. $\Theta$ and $\Pi=\nabla \Phi$ are canonical conjugate boson fields:

$$
[\Theta(x), \Pi(y)]=i \delta(x-y) .
$$

The zero modes of the charge and current density are respectively:

$$
\begin{aligned}
& \widehat{N}=N_{0}+\widehat{Q}=\int_{0}^{L} \rho(x) d x=N_{0}-\int_{0}^{L} \frac{1}{\sqrt{\pi}} \nabla \Phi d x, \\
& \widehat{J}=\int_{0}^{L} j(x) d x=\int_{0}^{L} \frac{1}{\sqrt{\pi}} \nabla \Theta(x) d x .
\end{aligned}
$$

$\widehat{Q}$ has integral eigenvalues as befits a charge operator; in the bosonization mapping, the charge quantization is taken into account by the topological quantization of the phase field $\Phi$. Similarly, since $\int_{0}^{L} j(x) d x$ is a closed line integral (around the LL), it is a quantized number: this is just the topological quantization of the superfluid phase; the normalization of the fields have been chosen so that $\widehat{J}$ is an integer. For fermions, $\widehat{Q}=N_{+}+N_{-}$and $\widehat{J}=$ $N_{+}-N_{-}$where $N_{+}$and $N_{-}$are respectively the (integral) number of (bare) electrons added to the ground state at the right and left Fermi points. The construction we have reviewed above is due to Haldane 34 .

Integrating the Fourier expansions of the charge and current density gives:

$$
\begin{aligned}
& \Theta(x)=\Theta_{0}+\frac{\sqrt{\pi}}{L} \widehat{J} x+\frac{1}{\sqrt{L}} \sum_{n \neq 0} \Theta_{n} \exp i \frac{2 \pi n}{L} x, \\
& \Phi(x)=\Phi_{0}-\frac{\sqrt{\pi}}{L} \widehat{Q} x+\frac{1}{\sqrt{L}} \sum_{n \neq 0} \Phi_{n} \exp i \frac{2 \pi n}{L} x .
\end{aligned}
$$

Note that these fields are not periodic: this allows for the above mentioned topological excitations. We demand that the boson or fermion operators are physical objects and be periodic on the ring: $\Psi_{B / F}(x)=\Psi_{B / F}(x+L)$; this then implies the following selection rules on the eigenvalues $Q$ and $J$ of the zero modes $\widehat{Q}$ and $\widehat{J}$ :
Bosons: J even integer,

Fermions: $Q-J$ even integer.

Both $Q$ and $J$ are integers. The zero modes are sometimes extracted from the definition of the fermion operator which defines the $U_{ \pm}$operators, first built by Heides reich and Haldane for the Tomonaga-Luttinger model 31 .8:

$$
U_{ \pm}=\exp i \sqrt{\pi}\left(\Theta_{0} \pm \Phi_{0}\right) .
$$

It will be useful to consider the commutation properties of the following operators:

$$
V_{\alpha, \beta}(x)=: \exp -i \sqrt{\pi}(\alpha \Theta(x)-\beta \Phi(x)): .
$$

Using Campbell-Haussdorf formula, one finds:

$$
\begin{aligned}
& V_{\alpha, \beta}(x) V_{\alpha, \beta}(y) \\
= & V_{\alpha, \beta}(y) V_{\alpha, \beta}(x) e^{-i \pi \alpha \beta \operatorname{sgn}(y-x)}
\end{aligned}
$$

where $\operatorname{sgn}(x)$ is the sign function, which shows in particular that $\Psi_{F}(x)$ is a fermionic operator. We define the exchange statistics of an operator per:

$$
\begin{aligned}
& O(x) O(y) \\
= & O(y) O(x) \exp -i \theta \operatorname{sgn}(y-x) .
\end{aligned}
$$

For instance $\theta=\pi$ for fermions.

\section{Excitations}

Until the work of Heidenreich 31 and subsequently of Haldane 8 , the only excitations considered in the gaussian model were the bosonic phonon (or plasmon 14 ) modes. But the hamiltonian contains a second part corresponding to the energies of states with non-zero charge or current with respect to the ground state. In reciprocal space, the gaussian hamiltonian becomes:

$$
\begin{aligned}
H_{B}= & \frac{u}{2} \sum_{q \neq 0} K^{-1} \Pi_{q} \Pi_{-q}+K q^{2} \Theta_{q} \Theta_{-q} \\
& +\frac{\pi u}{2 L}\left(\frac{\widehat{Q}^{2}}{K}+K \widehat{J}^{2}\right) .
\end{aligned}
$$

We have split the hamiltonian into the phonon part and the non-bosonic zero mode part. The first term can indeed be rewritten as:

$$
H_{\text {phonon }}=\sum_{q \neq 0} u|q|\left(b_{q}^{+} b_{q}+\frac{1}{2}\right)
$$

with the phonon operators:

$$
\begin{aligned}
b_{q} & =\sqrt{\frac{K|q|}{2}}\left(\Theta_{q}-\frac{q}{K|q|} \Phi_{q}\right), \\
b_{q}^{+} & =\sqrt{\frac{K|q|}{2}}\left(\Theta_{-q}-\frac{q}{K|q|} \Phi_{-q}\right) .
\end{aligned}
$$


The second term in the hamiltonian is standard in conformal field theory; it corresponds to finite size corrections to the energy when one adds particles or creates persistent currents in the Luttinger liquid. The corresponding states are built by means of Haldane's $U_{ \pm}$operators which act as ladder operators in Fock space 8 . This $(Q, J)$ part of the hamiltonian is often called in CFT a zero mode part. The corresponding excitations may however carry momentum. A non zero $J$ excitation creates indeed a persistent current with momentum $J k_{F}$. These states are therefore non-dispersive since their momentum may only assume the discrete values $J k_{F}$.

The spectrum of the hamiltonian results from a convolution of plasmon excitations and of these $(Q, J)$ excitations as is apparent in figures (11,2): two linear plasmon branches rise from each local minimum of the energy obtained for the zero-mode states $(Q, J)$. It is important to note that there are selection rules on the allowed values of $(Q, J)$, which refer back to the quantum statistics of the particles: as reviewed in the previous section, the gaussian model can be considered either for bosons or fermions, which results in different bosonization formulas. For bosons, $J$ is constrained to be an even integer while for fermions, $Q$ and $J$ must have the same parity. This then leads to two different spectra as can be seen from figures (1) and (2): for instance, for bosons the state $(Q=1, J=0)$ is available while it is forbidden for fermions; conversely $(Q=1, J=1)$ is available to fermions but not to bosons. Thus we have two different theories: the same hamiltonian leads to different properties depending on whether we consider a Fock space of bosons or a Fock space of fermions 34 . We will call the LL with bosonic (resp. fermionic) selection rules: the bosonic (resp. fermionic) LL. For the bosonic LL, as depicted in figure (11) the spectrum in arbitrary charge sectors has the same form but for a shift in energies: in the charge sector $Q$ one must add the constant $\pi u Q^{2} /(2 L)$ to the energy. The same energies are found for the fermionic LL in charge sectors for which $Q$ is an even integer, but if $Q$ is an odd integer there is a new spectrum with local minima at momenta $\pm k_{F}$ and not $k=0$ (figure 2).

In the rest of the paper we refer to this parametrization of the spectrum in terms of phonons and zero modes as the zero mode basis; this is to be distinguished from the quasiparticle basis which we will build later. A property which will prove crucial for the rest of the discussion is the fact that in the free-fermion case a quasiparticle basis exists as an alternative to the zero mode basis: instead of the zero modes basis, it is indeed possible to parametrize the spectrum in terms of the usual Landau quasiparticles. Below we show that a similar quasiparticle basis can be built in the interacting case. While fractional quasiparticles do occur in exactly solvable models (the holon, the spinon), scant contact had been made with the bosonization approach as mentioned earlier. In the low-energy limit, using the bosonization formalism, we will directly recover the fractional excitations predicted in Bethe Ansatz, with the advantage that the simplifica- tions brought by the low-energy limit will allow a complete characterization, giving for instance easy access to statistical phases.

\section{FRACTIONAL EXCITATIONS OF THE SPINLESS LUTTINGER LIQUID.}

This section is divided as follows: first, we discuss the property of chiral separation which is central to the physics of fractionalization; then, we exhibit fractional quasiparticles for the bosonic LL before turning to the fermionic LL for which we will find a different set of elementary excitations.

\section{A. Chiral separation.}

\section{Chiral vertex operators and fractionalization.}

The gaussian model is endowed with a very basic property which is that of chiral separation, i.e. we can split it into two commuting parts corresponding to right or left propagation of the fields. This is a property which is systematically used by CFT in the analysis of conformally invariant systems. Indeed:

$$
\begin{gathered}
H_{B}=\frac{u}{2} \int_{0}^{L} d x K^{-1} \Pi(x)^{2}+K(\nabla \Theta(x))^{2}, \\
\Rightarrow\left[\partial_{x}^{2}-\frac{1}{u^{2}} \partial_{t}^{2}\right] \Theta(x, t)=0
\end{gathered}
$$

More precisely we introduce the following chiral fields:

$$
\Theta_{ \pm}(x)=\Theta(x) \mp \frac{\Phi(x)}{K}
$$

which are related to the phonon operators by:

$$
\begin{aligned}
& q>0: b_{q}=\sqrt{\frac{K|q|}{2}} \Theta_{+, q}, \\
& q<0: b_{q}=\sqrt{\frac{K|q|}{2}} \Theta_{-, q} .
\end{aligned}
$$

In terms of these fields the hamiltonian becomes:

$$
\begin{aligned}
H_{B} & =H_{+}+H_{-} \\
H_{ \pm} & =\frac{u K}{4} \int_{0}^{L} d x:\left(\partial_{x} \Theta_{ \pm}(x)\right)^{2}: \\
& =\sum_{ \pm q>0} u|q|: b_{q}^{+} b_{q}:+\frac{\pi u}{L K}\left(\frac{\widehat{Q} \pm K \widehat{J}}{2}\right)^{2} .
\end{aligned}
$$

$H_{+}$only contains right-moving phonons and similarly for $H_{-}$with left-moving phonons. It is clear also that 
$\left[H_{+}, H_{-}\right]=0$. Let us show now that these fields $\Theta_{ \pm}$are chiral; they obey the equal-time commutation relations:

$$
\left[\Theta_{ \pm}(x), \mp \frac{K}{2} \partial_{y} \Theta_{ \pm}(y)\right]=i \delta(x-y),
$$

which implies that the momentum canonically conjugate to $\Theta_{ \pm}$is $\Pi_{\Theta_{ \pm}}=\mp \frac{K}{2} \partial_{x} \Theta_{ \pm}$. The equations of motions for these fields are:

$$
u \partial_{x} \Theta_{ \pm}=\mp \partial_{t} \Theta_{ \pm}
$$

Thus: $\Theta_{ \pm}(x, t)=\Theta_{ \pm}(x \mp u t)$ which means that we have chiral fields indeed. The superfluid phase has therefore been parametrized as: $\Theta(x, t)=$ $\frac{1}{2}\left[\Theta_{+}(x-u t)+\Theta_{-}(x+u t)\right]$.

One may define chiral density operators as well as the corresponding chiral charges as:

$$
\begin{aligned}
\rho_{ \pm}(x) & =\frac{1}{2 \sqrt{\pi}} \partial_{x} \Phi_{ \pm}(x)=\frac{\delta \rho(x) \pm K j(x)}{2} \\
\widehat{Q}_{ \pm} & =\frac{\widehat{Q} \pm K \widehat{J}}{2}
\end{aligned}
$$

Those chiral densities obey the anomalous (Kac-Moody) commutation relations:

$$
\left[\rho_{ \pm}(x), \rho_{ \pm}(y)\right]=\mp \frac{i K}{2 \pi} \partial_{x} \delta(x-y) .
$$

Let us now consider the injection of $Q$ particles with a momentum $q$ and current $J$. In that case, the plasmon total momentum is equal to $q-J\left(k_{F}+\frac{\pi Q}{L}\right)$. In the bosonization formalism, the operator creating this state is:

$$
V_{Q, J}(q)=\frac{1}{\sqrt{L}} \int_{0}^{L} d x e^{i\left(q-J k_{F}\right) x}: \exp -i \sqrt{\pi}(Q \Theta-J \Phi):
$$

This can also be rewritten as:

$$
\begin{aligned}
V_{Q, J}(q)= & \frac{1}{\sqrt{L}} \int_{0}^{L} d x e^{i\left(q-J k_{F}\right) x} \exp -i \sqrt{\pi} Q_{+} \Theta_{+}(x) \\
& \times \exp -i \sqrt{\pi} Q_{-} \Theta_{-}(x) .
\end{aligned}
$$

As a function of time:

$$
\begin{aligned}
V_{Q, J}(q, t)= & \frac{1}{\sqrt{L}} \int_{0}^{L} d x e^{i\left(q-J k_{F}\right) x} \exp -i \sqrt{\pi} Q_{+} \Theta_{+}(x-u t) \\
& \times \exp -i \sqrt{\pi} Q_{-} \Theta_{-}(x+u t) .
\end{aligned}
$$

There is therefore a splitting into two counterpropagating states. For non-interacting electrons the chiral charges $Q_{ \pm}$are integers since $K=1$ and the operators exp $-i \sqrt{\pi} Q_{ \pm} \Theta_{ \pm}(x \mp u t)$ are just those of $Q_{ \pm}$Landau quasiparticless. But in the general case this is not true anymore: we will therefore have states carrying fractional charges.
We now define the chiral vertex operators which appeared in the previous expression as:

$$
V_{Q_{ \pm}}^{ \pm}(x)=\exp -i \sqrt{\pi} Q_{ \pm} \Theta_{ \pm}(x),
$$

where the upperscript \pm refers to the direction of propagation. They obey the following commutation rules:

$$
\begin{aligned}
{\left[\rho(x), V_{Q_{ \pm}}^{ \pm}(y)\right] } & =Q_{ \pm} \delta(x-y) V_{Q_{ \pm}}^{ \pm}(x), \\
{\left[\widehat{Q}, V_{Q_{ \pm}}^{ \pm}(x)\right] } & =Q_{ \pm} V_{Q_{ \pm}}^{ \pm}(x), \\
{\left[\widehat{J}, V_{Q_{ \pm}}^{ \pm}(x)\right] } & =\frac{Q_{ \pm}}{K} V_{Q_{ \pm}}^{ \pm}(x),
\end{aligned}
$$

which shows they carry charges $Q_{ \pm}=\frac{Q \pm K J}{2}$ which are non-integral in general. The above operator identity means that the charge is 'sharp': by 'sharp' we mean that the charge found is not a quantum average $(\langle Q\rangle$ is not necessarily quantized of course). This is a point we want to stress because this means that these quantum states are genuinely fractional. This shows then that if one injects $Q$ particles with current $J$ in a LL, one should observe a charge $Q_{+}=\frac{Q+K J}{2}$ state propagating to the right at velocity $u$ and a charge $Q_{-}=\frac{Q-K J}{2}$ going to the left with velocity $-u$. For instance, let us inject an electron exactly at the right Fermi point: this is a $(Q=1, J=1)$ excitation (with no plasmon excited); there would then be fractionalization into a charge $\frac{1+K}{2}$ state going to the right and a charge $\frac{1-K}{2}$ going to the left.

The most important property of these fractional states is that they are exact eigenstates of the gaussian hamiltonian. The proof requires a proper definition of their Fourier transform because they are anyons, as will be shown shortly: from equation (1.22) it is clear indeed that the commutation relations are anyonic with an anyonic phase

$$
\theta= \pm \pi \frac{Q_{ \pm}^{2}}{K}
$$

Due to its anyonic character $V_{Q_{ \pm}}^{ \pm}(x)$ does not obey periodic boundary conditions; if we use the expressions of the fields $\Phi$ and $\Theta$ (equations 1.17, 1.16), we immediately find that:

$$
V_{Q_{ \pm}}^{ \pm}(x+L)=\exp \pm i 2 \pi \frac{Q_{ \pm}^{2}}{K} V_{Q_{ \pm}}^{ \pm}(x) .
$$

The Fourier transform is then defined as:

$V_{Q_{ \pm}}^{ \pm}\left(q_{n}\right)=\frac{1}{\sqrt{L}} \int_{0}^{L} d x \exp -i\left(\frac{2 \pi}{L} n \pm \frac{2 \pi}{L} \frac{Q_{ \pm}^{2}}{K}\right) x V_{Q_{ \pm}}^{ \pm}(x)$,

with a pseudo-momentum $q_{n}$ quantized as:

$$
\begin{aligned}
q_{n} & =\frac{2 \pi}{L} n \pm \frac{2 \pi}{L} \frac{Q_{ \pm}^{2}}{K} \\
& =\overline{q_{n}} \pm \frac{2 \pi}{L} \frac{Q_{ \pm}^{2}}{K}
\end{aligned}
$$


(where we have defined a phonon part $\overline{q_{n}}$ of the momentum).

The operators $V_{Q_{ \pm}}^{ \pm}\left(q_{n}\right)$ are such that:

1) $V_{Q_{ \pm}}^{ \pm}(q) \mid \Psi_{0}>$ is an exact eigenstate of the chiral hamiltonian $H_{ \pm}$with energy:

$$
E\left(Q_{ \pm}, \overline{q_{n}}\right)=\left[u\left|\overline{q_{n}}\right|+\frac{\pi u}{2 L} \frac{Q_{ \pm}^{2}}{K}\right] .
$$

where $\left|\Psi_{0}\right\rangle$ is the interacting ground state (see appendix). It has a linear dispersion.

2) The states created by the $V_{Q_{ \pm}}^{ \pm}\left(q_{n}\right)$ to which one adds the phonon excitations form a complete set. This is obripus because the states $V_{Q, J}(x)$ span the full Fock space 35 .

\section{The LL spectrum in terms of fractional quasiparticles.}

Let us consider figures (1, 2, which show the spectrum of the LL hamiltonian in various charge sectors and ask the following question: what happens when one adds $Q$ particles to the system (i.e. in the charge sector $Q$ )? In the standard view of the LL spectrum based on the phonon and zero modes basis, the dynamics of the charge added to the LL is unclear because it is concealed in the zero modes. The parametrization of the spectrum in terms of the zero modes and the phonons does not allow to find what happens once the charge $Q$ is added to the system because that choice of basis involves the use of non-dynamical states (Haldane's $U_{p m}$ operators, which describe the zero modes). By contrast the quasiparticle basis only involves states which have a dynamics (the phonons and the fractional states) and we are therefore able to tell what happens to the charge, how much of it will move to the right, and so forth: if we consider the two branches starting from $k=J k_{F}$ in the charge sector $Q$, the right branch corresponds to a right-moving fractional excitation with linear dispersion and with charge $Q_{+}=\frac{Q+K J}{2}$, while the left branch is due to a left-moving fractional state with charge $Q_{-}=\frac{Q-K J}{2}$. The continuum in between the branches simply results from the creation of the two fractional excitations with both non-zero momentum $\overline{q_{+, n}}$ and $\overline{q_{-, n}}$ (on the right branch, a charge $Q_{-}=\frac{Q-K J}{2}$ is also created but it has zero momentum $\overline{q_{-, n}}=0$, and conversely on the left branch).

The direct way to find out how the charge $Q$ will behave, is to exhibit the quantum states which will describe the propagation of the charge. This is what the quasiparticle basis does because it directly considers the states involved in the dynamics of the charge. Of course, the two bases (the quasiparticle basis and the zero mode basis) are mathematically equivalent and therefore lead to identical physics: therefore the charge dynamics can also in principle be determined in the zero mode basis, but in the quasiparticle basis, we have the benefit that the spectroscopy immediately tells us the fate of the charge added to the system. In sharp contrast, in the zero mode basis, the spectroscopy is not useful because the states used in that basis are the phonons (which have no charge) and the $U_{ \pm}$operators (which have charge but no dynamics). We will give such an argument in the next section: this will prove in an independent manner the fractionalization of the LL spectrum (in a way which does not depend on the explicit construction of the fractional states operators).

\section{Selection rules and fractionalization.}

The fractional charges carried by the fractional excitations considered above are not arbitrary: they must take on the values

$$
Q_{ \pm}=\frac{Q \pm K J}{2}
$$

where both $Q$ and $J$ are integers. We may view these constraints on the allowed spectrum of fractional charges as selection rules. These selection rules have however a clear physical meaning which we discuss now.

Although these excitations do not carry the electron quantum numbers because of the fractionalization of the spectrum, nevertheless the elementary constituents of our system are electrons (they are the high energy elementary particles of our systems): this means that they alone define the structure of Fock space, with the implication that all physical states must consist of an integrer number of electrons. Despite the fact that there are fractional states, the previous remark implies that these fractional states will be created in appropriate combinations so that the total charge is always an integer. This is the explanation of the previous selection rules we found, which in fine enforce the basic constraint that we started out with electrons. We may view these selection rules as being topological since they are directly related to the structure of the Fock space.

It is easy to show that eq. (2.27) immediately follows from the requirement that all states are electronic. We consider two counterpropagating states with arbitrary charge $Q_{+}$and $Q_{-}$; we make no hypothesis on the values of the charges, nor on the nature of the chiral states (we do not assume they correspond to $V_{Q_{+}}^{+}$and $V_{Q_{-}}^{-}$). The only assumptions we make are the following: (a) the one-dimensionality which means that the eigenstates have momenta in one of either two directions and (b) that the current density operator is renormalized. We then have two constraints on the values that the charges $Q_{+}$and $Q_{-}$may assume: since our Fock space is that of electrons, all the states contain an integer number of electrons i.e. $Q_{+}+Q_{-}=Q$ is an integer. The second constraint stems from the renormalization of the current density operator:

$$
j_{R}(x)=u K j(x)=u K\left(\frac{1}{\sqrt{\pi}} \partial_{x} \Theta(x)\right)
$$


where $j(x)$ is the current density in the non-interacting case. This expression can be derived from the continuity equations. Going around a ring of length $L$ in the LL we get a (persistent) current which must be quantized:

$$
J_{R}=\int_{0}^{L} d x j_{R}(x)=u K J
$$

where $J$ is an integer 36 ; but the current carried by the states with charges $Q_{+}$and $Q_{-}$is $J_{R}=u\left(Q_{+}-Q_{-}\right)$. Therefore:

$$
\left(Q_{+}-Q_{-}\right)=K J, J \text { integer }
$$

while:

$$
\left(Q_{+}+Q_{-}\right)=Q, Q \text { integer }
$$

Solving for these constraints, one recovers the selection rules (2.27), i.e the spectrum of fractional charges. We observe in passing that this argument does not depend on our formal algebraic derivation of subsection (II A 1) and provides an alternative proof of the existence of fractional states as well as it yields the allowed charge spectrum. In that argument, fractionalization follows from the renormalization of the current in the presence of interactions.

\section{B. Elementary excitations of the bosonic LL.}

\section{Elementary excitations.}

We now establish a series of new results concerning the elementary chiral excitations of a non-chiral LL. We would like to find a basis of elementary excitations, i.e. identify objects from which all the other excitations can be built. It will be useful to use a spinor notation to represent the fractional states:

$$
\left(\begin{array}{l}
Q_{+} \\
Q_{-}
\end{array}\right)=\left(\begin{array}{l}
\frac{Q+K J}{2} \\
\frac{Q-K J}{2}
\end{array}\right) .
$$

(2.32) should be understood as follows: the fractional state $V_{Q_{+}}^{+}$which is an anyon propagating with velocity $u$ is created along with the fractional state $V_{Q_{-}}^{-}$which propagates in the opposite direction with the velocity $-u$. The selection rules are encoded in the second spinor: the equation is then read as meaning that addition of $Q$ particles with (persistent) current $J$ will result in a splitting into the two counterpropagating fractional states $V_{Q_{+}}^{+}$ and $V_{Q_{-}}^{-}$.

We must carefully distinguish between Bose and Fermi statistics because of the constraints on $Q$ and $J$. Let us consider bosons first: since $J$ is even we can rewrite it as $J=2 n$ where $n$ is now an arbitrary integer. But then for bosons this implies that the spinor can be written in terms of two other independent spinors:

$$
\begin{aligned}
\left(\begin{array}{l}
Q_{+} \\
Q_{-}
\end{array}\right) & =\left(\begin{array}{l}
\frac{Q+K J}{2} \\
\frac{Q-K J}{2}
\end{array}\right) \\
& =Q\left(\begin{array}{l}
\frac{1}{2} \\
\frac{1}{2}
\end{array}\right)+n\left(\begin{array}{l}
K \\
-K
\end{array}\right) .
\end{aligned}
$$

This implies that in real space the fractional charge excitation is:

$$
V_{Q_{ \pm}}^{ \pm}(x)=\left[V_{1 / 2}^{ \pm}(x)\right]^{Q}\left[V_{ \pm K}^{ \pm}(x)\right]^{n},
$$

where $Q$ and $n$ are now independent integers of arbitrary sign: $(Q, n) \in Z^{2}$. In reciprocal space, one has a convolution for the exact fractional eigenstate:

$$
\begin{aligned}
V_{Q_{ \pm}}^{ \pm}(\bar{q})= & \int . . \int \prod_{i=1}^{Q} d \overline{q_{i}}\left[V_{1 / 2}^{ \pm}\left(\overline{q_{i}}\right)\right] \prod_{j=1}^{n} d \overline{p_{j}}\left[V_{ \pm K}^{ \pm}\left(\overline{p_{j}}\right)\right] \\
& \times \delta\left(\sum_{i=1}^{Q} \overline{q_{i}}+\sum_{j=1}^{n} \overline{p_{j}}-\bar{q}\right)
\end{aligned}
$$

(the momenta in that expression are the phonon parts $\overline{q_{n}}$ of the momentum of the operator: for $V_{Q_{ \pm}}^{ \pm}\left(q_{n}\right), q_{n}=$ $\overline{q_{n}} \pm \frac{2 \pi}{L} \frac{Q_{ \pm}^{2}}{K}$ and $\left.\overline{q_{n}}=\frac{2 \pi n}{L}\right) 37$.

The above equation demonstrates clearly that the excitation $V_{Q_{ \pm}}^{ \pm}(\bar{q})$ can be built from $Q$ charge $1 / 2$ states $V_{1 / 2}^{ \pm}$and $n$ charge $\pm K$ states $V_{ \pm K}^{ \pm}$. The whole spectrum of fractional excitations is thus built by repeated creation of $V_{1 / 2}^{ \pm}$and $V_{ \pm K}^{ \pm}$which means that they are the elementary excitations we were seeking. These two elementary excitations will be identified in the following as respectively the spinon (for spin systems) and a (1D) Laughlin quasiparticle.

\section{Wavefunctions of the fractional excitations.}

To be complete, we compute the wavefunctions of all the chiral excitations. We will first need the ground state wavefunction which is simply a Jastrow wavefunction: this is of course expected since the gaussian hamiltonian is the $1 \mathrm{D}$ version of the acoustic hamiltonian of Chester and Reatto's Jastrow theory of He 433 and is also identical to Bohm-Pines RPA plasmon hamiltonian adapted to 1 D 38 . Since the gaussian hamiltonian is a sum of oscillators, the ground state is a gaussian function of the densities:

$$
\begin{aligned}
& \Psi_{0, B}\left(\left\{\rho_{q}\right\}\right) \\
= & \exp \left(-\sum_{q \neq 0} \frac{\pi}{2 K|q|} \rho_{q} \rho_{-q}\right) \\
= & \exp \frac{1}{2 K} \iint d x d x^{\prime} \widehat{\rho}(x) \ln \left|\sin \frac{\pi}{L}\left(x-x^{\prime}\right)\right| \widehat{\rho}\left(x^{\prime}\right) .
\end{aligned}
$$

This expression is valid for the bosonic LL; for the fermionic LL, antisymmetry is recovered by observing 
that the fermionic LL simply derives from the bosonic LL through a singular gauge transformation on the bosonic LL (the Jordan-Wigner transformation) 39. this is exactly as in the composite boson Chern-Simons theory for which the hamiltonian is plasmon-like at the oneloop level (RPA) and whose ground state is of course symmetric (the modulus of Laughlin wavefunction); in that theory, the Laughlin state is then found after undoing the Chern-Simons gauge transformation 10 . Similarly undoing the Jordan-Wigner transformation amounts to multiplying the bosonic ground state by the phase factor $\prod_{i<j} \operatorname{sgn}\left(x_{i}-x_{j}\right)=\prod_{i<j}\left(\frac{\left(x_{i}-x_{j}\right)}{\left|x_{i}-x_{j}\right|}\right)$ (that phase factor is found by applying the Jordan-Wigner operator on the ground state). The wavefunction is the $1 \mathrm{D}$ analog of the 2D Laughlin state of FQHE if we rewrite the previous expression in terms of the particles' positions: $\rho(x)=\sum_{i} \delta\left(x-x_{i}\right)$, and by introducing the circular coordinates $z=\exp i \frac{2 \pi}{L} x$ :

$$
\psi_{0, B}\left(\left\{x_{i}\right\}\right)=\prod_{i<j}\left|z_{i}-z_{j}\right|^{1 / K}
$$

The wavefunctions of the excited states can now be computed 41. Let us consider first the operator $\exp -i \sqrt{\pi} \alpha \Theta\left(x_{0}\right)$; since $\Theta$ is the canonical conjugate of the field $\Pi=\partial_{x} \Phi=-\sqrt{\pi} \delta \widehat{\rho}$,

$$
\Theta(x)=-\frac{1}{i \sqrt{\pi}} \frac{\delta}{\delta \widehat{\rho}(x)},
$$

and therefore:

$$
\begin{gathered}
\exp -i \sqrt{\pi} \alpha \Theta\left(x_{0}\right) \mid \Psi_{0, B}> \\
=\exp \alpha \frac{\delta}{\delta \widehat{\rho}\left(x_{0}\right)} \\
\quad \times \exp \frac{1}{2 K} \iint d x d x^{\prime} \widehat{\rho}(x) \ln \left|\sin \frac{\pi}{L}\left(x-x^{\prime}\right)\right| \widehat{\rho}\left(x^{\prime}\right) \\
=\exp \frac{\alpha}{K} \int d x \widehat{\rho}(x) \ln \left|\sin \frac{\pi}{L}\left(x-x_{0}\right)\right| \Psi_{0, B} \\
=C \prod_{i}\left|z_{i}-z_{0}\right|^{\alpha / K} \prod_{i<j}\left|z_{i}-z_{j}\right|^{1 / K}
\end{gathered}
$$

where $C$ is an unessential constant.

Similarly:

$$
\begin{aligned}
& \exp \pm i \sqrt{\pi} \frac{Q_{ \pm}}{K} \Phi(x) \exp \mp i \frac{Q_{ \pm}}{K} k_{F} x \\
= & \exp \mp i \pi \frac{Q_{ \pm}}{K} \int_{0}^{x} \widehat{\rho}(y) d y \\
= & \exp \mp i \pi \frac{Q_{ \pm}}{K} \int_{0}^{L} \widehat{\rho}(y) \theta(x-y) d y \\
= & \prod_{i}\left[\frac{\left(x_{i}-x\right)}{\left|x_{i}-x\right|}\right]^{\mp Q_{ \pm} / K}
\end{aligned}
$$

$$
=\prod_{i}\left[\frac{\left(z_{i}-z\right)}{\left|z_{i}-z\right|}\right]^{\mp Q_{ \pm} / K} \exp \pm i k_{F} \frac{Q_{ \pm}}{K}\left(\frac{\sum_{i} x_{i}}{N_{0}}+x\right)
$$

where we use the definitions of $\rho$ and $z$ introduced above and where

$$
\theta(x)=\frac{1}{i \pi} \ln \left[\frac{-x}{|x|}\right]
$$

is the Heaviside step function.

The above operator can thus be seen as a generalized Jordan-Wigner operator, since it multiplies wavefunctions by a singular phase factor; in this manner we recover the phase $\prod_{i<j}\left(\frac{\left(x_{i}-x_{j}\right)}{\left|x_{i}-x_{j}\right|}\right)$ of the ground state of the fermionic LL. Finally we have that:

$$
\begin{aligned}
& V_{Q_{+}}^{+}(x) \Psi_{0, B}\left(x_{1}, . ., x_{N}\right) \\
= & C \prod_{i}\left(\overline{z_{i}}-\bar{z}\right)^{Q+/ K} \prod_{i<j}\left|z_{i}-z_{j}\right|^{1 / K} \\
& \times \exp i k_{F} \frac{Q_{+}}{2 K}\left(\frac{\sum_{i} x_{i}}{N_{0}}+x\right),
\end{aligned}
$$

with a similar expression for $V_{Q_{-}}^{-}$(the bar over $z$ denotes complex conjugation). It is noteworthy that these wavefunctions are obtained by multiplying a Jastrow ground state with a Laughlin-like prefactor $\prod_{i}\left|z_{i}-z\right|^{Q_{ \pm} / K}$ which generalizes the Laughlin quasihole factor $\prod_{i}\left(z_{i}-\right.$ $z)$. We can now write down the wavefunctions of the two elementary excitations:

$$
\begin{aligned}
& V_{1 / 2}^{+}(x) \Psi_{0, B}\left(x_{1}, . ., x_{N}\right) \\
= & C \prod_{i}\left(z_{i}-z\right)^{1 / 2 K} \prod_{i<j}\left|z_{i}-z_{j}\right|^{1 / K} \\
& \times \exp -i \frac{k_{F}}{2 K}\left(\frac{\sum_{i} x_{i}}{N_{0}}+x\right),
\end{aligned}
$$

and,

$$
\begin{aligned}
& V_{K}^{+}(x) \Psi_{0, B}\left(x_{1}, . ., x_{N}\right) \\
= & C \prod_{i}\left(z_{i}-z\right) \prod_{i<j}\left|z_{i}-z_{j}\right|^{1 / K} \\
& \times \exp -i k_{F}\left(\frac{\sum_{i} x_{i}}{N_{0}}+x\right) .
\end{aligned}
$$

We see that $V_{K}^{+}(x)$ is nothing but the $1 \mathrm{D}$ counterpart of the 2D Laughlin quasi-hole wavefunction, provided we make the following correspondence between 1D and 2D wavefunctions: $K \Longleftrightarrow \nu, z=\exp i 2 \pi x / L \Longleftrightarrow$ $z=x+i y$ (up to a galilean boost absorbing the factor $\left.\exp -i k_{F}\left(\frac{\sum_{i} x_{i}}{N_{0}}+x\right)\right)$ : in view of the formal analogy we will call that state a $1 D$ Laughlin state. 


\section{The spinon.}

We found an elementary excitation $V_{1 / 2}^{ \pm}(x)$ for the bosonic LL carrying a charge $1 / 2$. When we consider spins, which are hard-core bosons, this result translates into having a state carrying a spin $\Delta S_{z}=1 / 2$ with respect to the ground state. In spin language, adding a particle into the system $(Q=1)$ corresponds to flipping a spin $\left(\Delta S_{z}=1\right)$. But it follows from eq.(2.33) that this excitation is a composite of two elementary excitations, each carrying a charge $1 / 2$. Therefore a pair of states with spin $S_{z}=1 / 2$ is created when one flips a spin $\left(\Delta S_{z}=1\right)$. We naturally identify this fractional spin excitation as a spinon. The spinon can be generated without any Laughlin quasiparticless if the spin current is zero $(J=0)$ : this is a process which we term a pure spin process, to be distinguished from a pure spin current process $\left(S_{z}=0\right)$ which generates Laughlin quasiparticlequasihole pairs (see below).

The properties of the spinon specifically depend on the LL parameter $K$. Although the spin is always $S_{z}=1 / 2$ the exchange statistics varies continuously with $K$ (i.e. when one varies the interaction):

$$
\theta_{\text {spinon }}=\frac{\pi}{4 K} \text {. }
$$

For instance for $K=1 / 2$ (which corresponds to $S U(2)$ symmetric spin interactions) the spinon is a semion. In that special case, the spinon wavefunction we obtain coincides exactly with that proposed by Haldane for the Haldane-Shastry spin chain²:

$$
\begin{aligned}
\Psi_{\text {spinon }}(z)= & \prod_{i}\left(z_{i}-z\right) \prod_{i<j}\left|z_{i}-z_{j}\right|^{2} \\
& \times \exp -i k_{F}\left(\frac{\sum_{i} x_{i}}{N_{0}}+x\right)
\end{aligned}
$$

In this expression the coordinates are those of the down spins. For $K=1 / 2$ the spinon and the spin $K$ Laughlin quasiparticles are identical. Although we have discussed fractional excitations for spin systems, the previous considerations apply of course to bosons: the "spinon" is then a charge $1 / 2$ excitation. For convenience we will call the excitation a spinon even when we consider bosons.

\section{The LL Laughlin quasiparticle.}

The second elementary excitation we found has the following wavefunction:

$$
\begin{aligned}
\Psi_{\text {Laughlin-qp }}\left(z_{0}\right)= & \prod_{i}\left(z_{i}-z_{0}\right) \prod_{i<j}\left|z_{i}-z_{j}\right|^{1 / K} \\
& \times \exp -i k_{F}\left(\frac{\sum_{i} x_{i}}{N_{0}}+x_{0}\right),
\end{aligned}
$$

which leads us to identify it with a Laughlin quasiparticles. The parallels which can be drawn between the $2 \mathrm{D}$
Laughlin quasi-hole and the Luttinger liquid Laughlin quasiparticles are indeed very strong. For instance as in $2 \mathrm{D}$ one can use the plasma analogy to find the fractional charge:

$$
\begin{aligned}
& \left|\Psi_{\text {Laughlin-qp }}\left(z_{0}\right)\right|^{2}=\left|\prod_{i}\left(z_{i}-z_{0}\right) \prod_{i<j}\right| z_{i}-\left.\left.z_{j}\right|^{1 / K}\right|^{2} \\
= & \exp \frac{1}{K} \iint d x d x^{\prime}\left[\hat{\rho}(x)+K \delta\left(x-x_{0}\right)\right] \\
& \ln \left|\sin \frac{\pi}{L}\left(x-x^{\prime}\right)\right|\left[\widehat{\rho}\left(x^{\prime}\right)+K \delta\left(x^{\prime}-x_{0}\right)\right] .
\end{aligned}
$$

The above expression clearly shows that the charge carried by the excitation is $K$ in agreement with the direct algebraic determination (using the operator $V_{K}$ ). There are however several differences between the LL Laughlin quasiparticles and its $2 \mathrm{D}$ famous counter-part; first, there is no analyticity requirement in the $1 \mathrm{D}$ problem, since we do not have to project into the lowest Landau level: we have two chiralities and the LL Laughlin quasi-electron is simply

$$
\begin{aligned}
\Psi_{\text {Laughlin-qe }}\left(z_{0}\right)= & \prod_{i}\left(z_{i}-z_{0}\right)^{-1} \prod_{i<j}\left|z_{i}-z_{j}\right|^{1 / K} \\
& \times \exp -i k_{F}\left(\frac{\sum_{i} x_{i}}{N_{0}}+x_{0}\right) .
\end{aligned}
$$

Second, while topological quantization forces the 2D FQHE Laughlin quasiparticles to have a rational charge, the charge of the 1D LL Laughlin quasiparticles can take on arbitrary real positive values, in particular irrational. This is a very startling property: irrational spin had already been proposed for solitens in coexisting CDWSDW systems by B. Horowith 2 , but in a sense this is perhaps less surprising since in one dimension there is no quantization axis for spin which can therefore take a continuum of values. We show below that the Laughlin quasiparticles also exist for the fermionic LL; furthermore we will find that for the fermionic LL there is another elementary excitation which may have an irrational charge.

How are Laughlin quasiparticles created in a LL? They are generated whenever $J \neq 0$; they are always created as quasiparticle-quasihole pairs. In particular in pure current processes $(Q=0)$ no "spinon" is created and we have only Laughlin quasiparticle-quasihole (qp-qh) pairs. For a persistent current $J$ excitation with $Q=0$ it follows from the expression $Q_{ \pm}=\frac{Q \pm K J}{2}$ that $J / 2$ quasiparticlequasihole pairs are generated.

From the above analysis we now can give a physical interpretation to the renormalization of the current density operator in the presence of interactions:

$$
\begin{aligned}
j(x) & =\frac{1}{\sqrt{\pi}} \partial_{x} \Theta(x), \\
& \longrightarrow j_{R}(x)=\frac{u K}{\sqrt{\pi}} \partial_{x} \Theta(x) .
\end{aligned}
$$


The velocity $u$ has been normalized to the Fermi velocity so that $u=1$ in the absence of interactions for fermions $(K=1)$. We have found that current excitations were due to Laughlin quasiparticles. The natural explanation of the renormalization is therefore that the current is no longer carried by Landau quasiparticles but by Laughlin quasiparticles with velocity $u$ and charge $K$.

\section{The bosonic LL spectrum in terms of fractional elementary excitations.}

For the bosonic LL we can now add the following precisions to the description of the spectrum.

For $Q=0$ excitations (see figure (11)), the continuum is due to multiple Laughlin quasiparticle-quasihole pairs: the right branch starting at $k=2 k_{F}$ corresponds to the propagation of a 1D Laughlin quasielectron while the left branch is due to a Laughlin quasihole; in between the two lines, we have a continuum generated by these two excitations. More generally at $k=2 n k_{F}$ where $n$ is an arbitrary integer, the two branches create a continuum of $n$ Laughlin quasiparticle-quasihole pairs. (Note that for $n=0$, which is an exceptional case, we have multiphonon processes.) Therefore the spectrum in the zero charge sector is not a Landau quasiparticle-quasihole pair continuum except at the special value $K=1$ which describes indeed in the low energy limit a gas of hard-core bosons.

In the charge sector $Q=1$, pairs of charge one-half excitations are created: they correspond to the "spinons" of spin chains; the pairs are superimposed on the previous Laughlin quasiparticle continuum: for instance a $2 k_{F}$ excitation generates a Laughlin quasiparticle-quasihole pair in addition to the "spinon" pair.

The Laughlin quasiparticle and the spinon are dual states for the bosonic LL; by duality we mean electromagnetic duality which exchanges charge and current processes. Indeed the "spinon" is associated with charge processes while the Laughlin quasiparticles is due to current excitations. The duality operation which maps a bosonic LL onto another bosonic LL is: $K=1 /\left(4 K^{\prime}\right)$ with $\Theta=2 \Phi^{\prime}$ and $\Phi=\Theta^{\prime} / 2$; zero modes then transform as $J=2 Q^{\prime}$ et $Q=J^{\prime} / 2$. With these relations, the selection rule remains bosonic $\left(J^{\prime}\right.$ even) while the hamiltonian $H_{B}[K, \Theta, \Phi]=H_{B}\left[K^{\prime}, \Theta^{\prime}, \Phi^{\prime}\right]$ retains a gaussian form. It is clear then that $K=1 / 2$ is a self-dual point while $V_{K}$ and $V_{1 / 2}$ create dual quasiparticles. This is not true for the fermionic LL.

\section{The $X X Z$ spin chain.}

Let us illustrate these results on the specific example of the anisotropic Heisenberg $X X Z$ spin chain. The hamiltonian of the $X X Z$ spin chain with anisotropy $\Delta$, after a bipartite rotation is:
$H[J, \Delta]=J \sum_{i}\left\{-\frac{1}{2}\left(S_{i}^{+} S_{i+1}^{-}+S_{i}^{-} S_{i+1}^{+}\right)+\Delta S_{i}^{z} S_{i+1}^{z}\right\}$.

As $\Delta$ is varied, one finds three phases: i) for $\Delta>1$ one gets an Ising antiferromagnet the twofold degenerate groundstate of which leads to solitonic excitations with spin one-half $1 / 2$ domain walls; ii) for $\Delta<1$ one has an Ising ferromagnet; iii) for $-1 \leq \Delta \leq 1$ we have the socalled $X Y$ phase: this is the Luttinger liquid phase we are interested in. The isotropic Heisenberg chain with $S U(2)$ invariance corresponds to $\Delta=1$. The Luttinger liquid parameter was determined exactly by Luther and Peschel on the basis of a comparison with the Baxter model43:

$$
K(\Delta)=\frac{\pi}{2 \arccos (-\Delta)} .
$$

The spectrum in the sector $\Delta S_{z}=1$ is shown in figure (3) for the Heisenberg model; its linearization through bosonization is also shown in figure.

Given thata spin one-half can be mapped onto a hardcore boson: 44 through the Holstein-Primakov transformation, we can transpose the results we found for the bosonic LL to the $X X Z$ spin chain.

If we want to compare the bosonization linearized spectrum to the exact one there are however two provisos: (a) we have to shift the bosonization spectrum by a momentum $\pi$ : this is due to the bipartite transformation one makes in the bosonization of the $X X Z$ spin chain (in order to change the sign of the $X Y$ term) and (b), there is a Brillouin zone: therefore we have to identify momenta modulo $2 \pi$ and since the Fermi vector is $k_{F}=\pi / 2$, excitations with $J / 2$ odd (resp. even) correspond to the same harmonics $k=\pi+J k_{F} \equiv 0$ (resp. $\pi$ ). Taking (a) and (b) into account, we can use the results of the previous section pertaining to the bosonic LL, to recover the linearized spectrum of the $X X Z$ chain.

We first consider the spin sector $\Delta S_{z}=0$. In figure (3) starting from momentum $\pi$ we have two straight lines corresponding to left and right moving phonons, bounding a continuum; due to the folding of the continuum spectrum of the bosonic LL, one superimposes on these lines the lines due to the creation of any even number of Laughlin qp-qh pairs (the qp dispersion being given by one line, and that of the qh by the other; if the qp is righthanded, its dispersion is that of the right line, etc...). Similarly the lines starting from momentum zero or $2 \pi$ correspond to the creation of an odd number of Laughlin qp-qh pairs. The continuum is therefore seen to be parametrized entirely in terms of the phonons and Laughlin quasiparticle-quasihole pairs while the zero mode basis relies on phonons and zero modes. The $\Delta S_{z}=1$ continuum is described in a similar manner but for the substitution of the phonons by a pair of counterpropagating spinons. In the special case of $S U(2)$ symmetry, the Laughlin quasiparticle and the spinon become identical 
operators. The previous parametrization reduces then to one involving only pairs of spinons because a pair of counterpropagating spinon plus a pair of counterpropagating spinon-antispinon is equivalent to a pair of spinons propagating in arbitrary directions. One then recovers the Bethe Ansatz result.

In the low-energy limit, we can now answer the various questions raised in the introduction about the spectrum of the $X X Z$ chain:

-what is the nature of the $\Delta S_{z}=1$ continuum? It is indeed a spinon pair continuum; but superimposed on them Laughlin quasiparticle-quasihole pairs can exist. The spinon changes when the anisotropy is varied: it acquires a statistical phase $\pi / 4 K=\arccos (-\Delta) / 2$. Therefore the spinons at different anisotropy are not adiabatically connected: they are orthogonal states, 45 this is consistent with numerical computations of the spectral density of the $S U(2)$ spinon, where it is found that the $S U(2)$ spinon has a zero quasiparticles weight for the $X Y$ chain 4 .

-what is the nature of the $\Delta S_{z}=0$ continuum? It is a Laughlin qp-qh pair continuum with an unquantized spin $S_{z}= \pm K= \pm \pi /(2 \arccos (-\Delta))$; in the $S U(2)$ symmetric case, they are identical to the spinons. In the $X Y$ limit, one recovers the standard spin one continuum predicted through a Jordan-Wigner transformation ( $K=1$, $\left.S_{z}= \pm 1\right)$. But in between these two points, the elementary excitation is neither a spinon nor a Jordan-Wigner fermion.

\section{The fermionic Luttinger Liquid.}

\section{Elementary excitations : the Laughlin quasiparticles and the "hybrid state".}

We now turn to fermions; the analysis of the elementary excitations will differ from that found for the bosonic LL because the allowed $(Q, J)$ states obey different selection rules, namely $J$ is not constrained any more to be an even integer, but must have the same parity as $Q$. We may therefore write $Q-J=2 n$. Then for fermions using eq.2.27:

$$
\left(Q_{+}, Q_{-}\right)=Q\left(\frac{1+K}{2}, \frac{1-K}{2}\right)-n(K,-K) .
$$

The most general excitation once again, is built by applying $Q$ times $V_{\frac{1 \pm K}{2}}^{ \pm}$and/or $n$ times $V_{ \pm K}^{ \pm}$to the ground state ; this means that we have identified a set of elementary excitations for the fermionic LL. Here too we find Laughlin quasiparticles $V_{ \pm K}^{ \pm}$, but instead of the spinon we get a "hybrid state": this is a consequence of statistics; as we will show below, that hybrid state is self-dual and is intermediate between the Laughlin quasiparticle and its dual state.

The Laughlin quasiparticle is created by current excitations: for a pure current process $(Q=0, J \neq 0)$ one indeed generates Laughlin qp-qh pairs as the above equation shows. The continuum for zero charge excitations $(Q=0)$ is often depicted as a (Landau) particle-hole continuum as in the non-interacting system $(K=1)$ : this is incorrect; we have instead a Laughlin quasiparticlequasihole continuum. The latter does reduce to the standard Landau quasiparticle continuum when $K=1$. For $k=J k_{F}$ there is a local minimum of the energy from which two linear branches rise corresponding to $J / 2=-n$ pairs of Laughlin quasiparticles and quasiholes. For the fermionic LL, the Laughlin quasiparticle is not the only state which may have an irrational charge: this is also possible for the hybrid state.

The hybrid state is created in mixed charge and current processes: this is the main difference with the bosonic LL for which the decoupling between charge and current processes is complete. As reviewed in the introduction, there are even-odd effects in the fermionic spectrum: the spectra obtained by adding an even or an odd number of particles are qualitatively different for the fermionic LL. The $Q=1$ continuum is understood as follows: the two branches at $k_{F}$ correspond to a pair of hybrid excitations carrying a charge $\frac{1-K}{2}$ and $\frac{1+K}{2}$, and propagating with velocities respectively $-u$ and $u$. At $-k_{F}$ the correspondence is reversed. More generally at $J k_{F}(J$ is an odd integer if $Q=1$ ), in addition to the hybrid quasiparticles, one also creates $J / 2-Q$ pairs of Laughlin quasiparticle and quasihole. When $K=1$ the hybrid states reduce to Landau quasiparticles. It is interesting to note an evidence for these states in the work of Safi and Schulz who considered the evolution of a charge 1 wavepacket injected at $k_{F}$ in a LL: they found that there was a splitting with an average charge $\langle Q\rangle=(1+K) / 2$ propagating to the right and an average charge $\langle Q\rangle=(1-K) / 2$ going to the left 47 . This is exactly what we predict. Note however a crucial difference: the charge they find is a quantum average while we deal with elementary excitations (exact eigenstates); this has an important consequence: while it is clear that on average a charge may assume irrational values, our result goes beyond that observation since it proves that there may exist in condensed matter systems a genuine good quantum state with sharp irrational charge.

In this section, we have found that for the fermionic LL there are two elementary excitations. One is the Laughlin quasiparticle already found for the bosonic LL. The second one is a hybrid state intermediate between the spinon and the Laughlin quasiparticle. The excitations corresponding to $Q=0$ transitions (they are particle-hole excitations in the non-interacting case), form a Laughlin quasiparticle-quasihole pair continuum when $K \neq 1$.

\section{Dual basis and the dual quasiparticles.}

The elementary excitations we have derived form a basis from which all the LL spectrum is recovered; by no 
means is this choice of basis unique: other bases of elementary excitations are generated with matrices associated with a basis change having integer entries whose inverses are also integer-valued: this ensures that all excitations are integral linear combinations of the elementary excitations. (The matrices belong to $S L(2, Z)$.) For instance for fermions, another basis of elementary excitations consists of states $V_{\frac{1 \pm K}{2}}^{ \pm}$and $V_{1}^{ \pm}$:

$$
\left(Q_{+}, Q_{-}\right)=J\left(\frac{1+K}{2}, \frac{1-K}{2}\right)+n(1,1) .
$$

It is actually a dual basis to the previous one: for fermions the electro-magnetic duality which exchanges charge and current excitations is expressed by $K \longleftrightarrow$ $1 / K$ and $\Phi \longleftrightarrow \Theta$. This is a canonical transformation; it results in: $H_{B}[K, \Theta, \Phi]=H_{B}[1 / K, \Phi, \Theta]$. The fermionic selection rule $Q-J$ even is obviously preserved: the transformation is therefore a duality operation for the fermionic LL. Observe that the transformations differ from those for the bosonic LL.

What is the nature of the elementary excitation $V_{1}^{ \pm}$? Under the duality transformation $V_{K}^{ \pm} \longrightarrow V_{-1}^{ \pm}$and $V_{-K}^{ \pm} \longrightarrow V_{1}^{ \pm}$. Therefore $V_{1}^{ \pm}$is an excitation dual to the Laughlin quasiparticle. It carries a charge unity and its wavefunction is:

$$
V_{1}^{+}\left(z_{0}\right) \Psi_{F}=\prod_{i}\left(z_{i}-z_{0}\right)^{1 / K} \prod_{i<j}\left|z_{i}-z_{j}\right|^{1 / K} \prod_{i<j} \frac{\left(z_{i}-z_{j}\right)}{\left|z_{i}-z_{j}\right|}
$$

We stress that although $V_{1}^{ \pm}$carries a unit charge, it is not an electron: the statistical exchange phase is $\pi / K$ which means $V_{1}^{ \pm}$is an anyon (it can be a fermion, in the special case $K=1 /(2 n+1))$. The difference with the electron is quite clear since the electron creation operator is:

$$
\begin{array}{r}
\Psi(x)=\sum_{n} \exp i(2 n+1) k_{F} x \\
\exp i(\sqrt{\pi} \Theta(x)-\sqrt{\pi}(2 n+1) \Phi(x)),
\end{array}
$$

while:

$$
V_{1}^{+}(x)=: \exp i \sqrt{\pi}\left(\Theta(x)-\frac{\Phi(x)}{K}\right):
$$

For $K \neq 1 /(2 n+1)$ the dual excitation appears to be a non-linear soliton of the electron. This excitation is interesting in many respects. If $K=1 /(2 n+1)$ (the Laughlin fractions) the excitation is fermionic and the exchange statistics of the operator is $\pi(2 n+1)$. The dual quasiparticle corresponds then to a sub-dominant harmonic of the electron Fourier expansion around $k \simeq(2 n+1) k_{F}$. If one attaches $2 n$ flux tubes to the electron (i.e. multiplies the electron operator by the Jordan-Wigner phase $\exp i \sqrt{\pi} 2 n \Phi)$ the dual state becomes the dominant $k=$
$k_{F}$ harmonics: this is exactly the composite fermion construction and it may then be more fitting to speak of a composite fermion (indeed, the statistics of the operator is $(2 n+1) \pi$ and not $\pi)$. Because of the similar long distance behavior of their Green functions, Stone proposed to identify such a sub-dominant operator -which he calls a hyperfermion-with Wen's electron operator introduced in the chiral LL 48 . This hyperfermion is identical to the dual state for $K=1 /(2 n+1)$. In general the dual state and the electron are however orthogonal: this is quite clear when one considers the LL with spin. The dual state is then generalized to a state with the same quantum numbers as the electron (carrying a unit charge and a spin one-half), but with again anyonic statistics. But due to spin-charge separation, that state is not stable and decays into a spinless charge one quasiparticle which is none other than the holon, and a spin one-half excitation, which is just the spinon. The dual excitation we have found is therefore the analog of the spinon and the holon for the spinless LL and has nothing to do (in general) with an electron. In the following in accordance with the previous remarks we will call these states holons (for the spinless LL) or dual states.

These dual holon states also occur in Haldane's interpretation of the Calogero-Sutherland model: he proposed that a natural interpretation of such a model was not in terms of electrons or bosons but as a gas of noninteracting anyon, 22, 27. The basis for that interpretation is the finding that for rational values of the coupling $\lambda=p / q$ ( $\lambda$ is related to the LL parameter by the simple relation $\lambda=1 / K)$, the dynamical structure factor obeys simple selection rules: $q$ "charge -1 bare particles" (the anyon -it has anyonic statistics $\pi \lambda=\pi / K$ ) are created with $p$ "holes" which therefore carry a charge $1 / \lambda$. The particles and holes appear as pseudo-particles in a pseudo-momenta parametrization of the spectrum. There are however difficulties with that interpretation: it is not clear how the selection rules are generalized when the coupling $\lambda$ is irrational; the physics is indeed completely continuous with the coupling while the selection rules are only valid for rational couplings; besides, an asymmetry is introduced between particles and holes. This means that this parametrization which relies on pseudoparticles is probably inadequate. In the low energy limit, the Calogero-Sutherland model has the properties of a Luttinger liquid. It is therefore possible to describe its quasiparticle spectrum in terms of the fractional excitations we have found in this paper: we do find the charge 1 anyon proposed (this is our dual state); however our selection rules are quite different. First they depend on the statistics (electrons or bosons): in contrast the pseudo-particle based selection rules do not involve spinons; this runs contrary to results on the bosonic LL for which the dual state must actually be seen as a composite state made out of two spinons. Second, our selection rules are valid even for irrational couplings that is imply the existence of quantum states with sharp irrational charges. Third our selection rules respect 
particle-hole duality: there exist both a charge one anyon with statistics $\pi / K$ (the dual holon) and a similar charge -1 anyon; the same applies to Laughlin quasiparticle for which we have both quasiholes and quasielectrons. Our selection rules involve the hybrid state and the Laughlin quasiparticles, and the holon only appears in the dual basis where it is accompanied again by the hybrid quasiparticle.

Finally, a symmetric basis can be associated with the hybrid excitation:

$$
\left(\begin{array}{l}
Q_{+} \\
Q_{-}
\end{array}\right)=m\left(\begin{array}{l}
\frac{1+K}{1-K} \\
\frac{1-}{2}
\end{array}\right)+n\left(\begin{array}{l}
\frac{1-K}{2} \\
\frac{1+K}{2}
\end{array}\right)
$$

where $m=\frac{Q+J}{2}$ and $n=\frac{Q-J}{2} ; m$ and $n$ are again independent integers. They physically correspond to the number of electrons added to the system at the right and left Fermi points respectively. That self-dual basis reduce to Landau quasiparticles when $K=1$. The physical processes generated in that symmetric basis are not charge or current excitations but addition of electrons at the Fermi surface. Note that the arbitrariness in the choice of a basis simply reflects the possibility to stress various specific physical processes as elementary. But experiments probe $Q_{+}$and $Q_{-}$; for a given set of $Q$ and $J, Q_{ \pm}$assume the same value irrespective of the basis choice.

\section{THE LUTTINGER LIQUID WITH SPIN.}

In this section we generalize the construction of fractional excitations developed in section (III) to the full Luttinger liquid with spin. One of the main properties exhibited by the effective theory is spin-charge separation, the complete decoupling of spin and charge dynamics. In the exact solution of the Hubbard model by Bethe Ansatz, excitations display such a spin-charge separation: one state, the holon is a spinless particle carrying the charge of the electron, while the other, the spinon, is a neutral spin one-half state 5 . This is an asymptotic property only valid in the low-energy limit (the Hubbard model in the large $U$ limit is an exception because spin-charge separation is realized at all energy scales). However this property is not obtained for all the gapless itinerant 1D models, in the low energy limit. According to the universality hypothesis they should be described by the Luttinger liquid framework if the interaction is not too long-ranged. One such example is the Hubbard model in a magnetic field which does not display spin-charge separation even in the low-energy limit, although it is a short range gapless model. This model was analyzed by Frahm and Korepin in the framework of Bethe Ansatz plus conformal field theory 25: they were able to compute the anomalous exponents for the correlation functions. Several issues remain unclear for such models in a magnetic field: in particular what the excitations are. Since spin-charge separation does not occur, the holon and the spinon cannot be the elementary excitations of the system anymore. To answer the question we have to turn to the low-energy effective theory. Frahm and Korepin's results imply that an effective description in terms of the gaussian model should be possible since conformal invariance is realized. We will find a generalization of the spin-charge separated gaussian hamiltonian suitable for a description of the Hubbard model in a magnetic field. Our formalism is very similar to Wen's $K$ matrix approach to edge states of the FQHE. This will enable us to characterize very precisely, in the lowenergy limit, the properties of 1D gapless models with or without spin-charge separation such as the Hubbard model in a magnetic field: we will find that in the latter case, although there is no spin-charge separation, there is still a generalized decoupling. The excitations are again fractional; as expected the holon and the spinon are no longer present in the spectrum and we will give the general framework allowing the description of the fractional states which replace them.

\section{A. Spin-charge separated Luttinger liquid.}

We start with the standard case when spin-charge separation exists. Although fractional excitations are clearly present in Bethe Ansatz, no description of these special states was attempted in the low-energy limit through bosonization. In the following we answer several questions: how does the holon evolve with interaction? What would be an effective wavefunction for it? Is it a semion? First, we consider the ground state of the two-component gaussian model, because it will suggest to us a possible generalization of the gaussian model which will prove to be the correct one for the description of gapless models without spin-charge separation such as the Hubbard model in a magnetic field.

\section{Ground state of the gaussian hamiltonian.}

We consider a two component model by introducing an internal quantum number such as the $S U(2)$ spin. We consider the charge and spin densities as well as their associated phase fields:

$$
\begin{aligned}
\rho_{c} & =\rho_{\uparrow}+\rho_{\downarrow} ; \rho_{s}=\rho_{\uparrow}-\rho_{\downarrow}, \\
\rho_{\sigma} & =-\frac{1}{\sqrt{\pi}} \partial_{x} \Phi_{\sigma}, \sigma=\uparrow, \downarrow \\
{\left[\Theta_{\sigma}(x), \partial_{x} \Phi_{\sigma^{\prime}}(y)\right] } & =i \delta_{\sigma \sigma^{\prime}} \delta(x-y), \\
\Phi_{c / s} & =\frac{\Phi_{\uparrow} \pm \Phi_{\downarrow}}{\sqrt{2}}, \\
{\left[\Theta_{\tau}(x), \partial_{x} \Phi_{\tau^{\prime}}(y)\right] } & =i \delta_{\tau \tau^{\prime}} \delta(x-y) ; \tau=c, s .
\end{aligned}
$$

The effective hamiltonian derived for instance from the Hubbard model in the absence of a magnetic field is: 


$$
\begin{aligned}
H & =H_{c}+H_{s}, \\
H_{\tau} & =\frac{u_{\tau}}{2} \int_{0}^{L} d x K_{\tau}^{-1}\left(\nabla \Phi_{\tau}\right)^{2}+K_{\tau}\left(\nabla \Theta_{\tau}\right)^{2} ; \tau=c, s .
\end{aligned}
$$

$\nabla \Phi_{\tau}=\Pi_{\tau}$ is canonically conjugate to the field $\Theta_{\tau}$. One easily extracts the ground state which is simply a product of gaussians:

$$
\Psi_{0}\left(\left\{\Pi_{\tau, q_{n}}\right\}\right)=\prod_{\tau=c, s} \exp \left(-\frac{1}{2 K_{\tau}} \sum_{n \neq 0} \frac{1}{\left|q_{n}\right|} \Pi_{\tau, q_{n}} \Pi_{\tau,-q_{n}}\right) .
$$

In terms of charge and spin densities:

$$
\Psi_{0}\left(\left\{\rho_{\tau, q_{n}}\right\}\right)=\prod_{\tau=c, s} \exp \left(-\frac{1}{4 K_{\tau}} \sum_{n \neq 0} \frac{\pi}{\left|q_{n}\right|} \rho_{\tau, q_{n}} \rho_{\tau,-q_{n}}\right) .
$$

The ground state displays of course a complete decoupling of spin and charge as is apparent from the previous expression. This is also a Jastrow wavefunction. In real space:

$$
\begin{gathered}
\Psi_{0}\left(\rho_{\tau}\right)=\prod_{\tau=c, s} \exp \frac{1}{4 K_{\tau}}[ \\
\left.\iint d x d x^{\prime} \rho_{\tau}(x) \ln \left|\sin \frac{\pi\left(x-x^{\prime}\right)}{L}\right| \rho_{\tau}\left(x^{\prime}\right)\right] .
\end{gathered}
$$

We define the charge and spin parts of the ground state per:

$$
\begin{aligned}
\Psi_{c / s}= & \exp \frac{1}{4 K_{c / s}}[ \\
& \left.\iint d x d x^{\prime} \rho_{c / s}(x) \ln \left|\sin \frac{\pi\left(x-x^{\prime}\right)}{L}\right| \rho_{c / s}\left(x^{\prime}\right)\right] .
\end{aligned}
$$

The previous ground state may be rewritten in terms of the densities of each species:

$$
\begin{gathered}
\Psi_{0}\left(\left\{\rho_{\sigma}\right\}\right)=\exp \frac{1}{2}[ \\
\left.\iint d x d x^{\prime} \rho_{\sigma}(x) g_{\sigma \sigma^{\prime}} \ln \left|\sin \frac{\pi\left(x-x^{\prime}\right)}{L}\right| \rho_{\sigma^{\prime}}\left(x^{\prime}\right)\right]
\end{gathered}
$$

where we have introduced the following $\widehat{g}$ matrix:

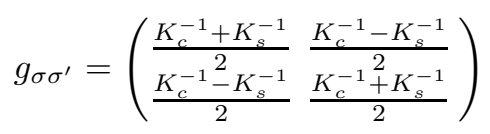

The eigenvalues of that matrix are simply the inverses of the Luttinger liquid parameters $K_{c}^{-1}$ and $K_{s}^{-1}$. If one rewrites the wavefunction in terms of individual electron coordinates $\rho_{\sigma}(x)=\sum_{i, \sigma} \delta\left(x-x_{i}\right)$ (the sum is restricted to particles with spin $\sigma$ ) and if one sets $z=\exp i \frac{2 \pi}{L} x$, one easily finds:

$$
\Psi_{0}\left(\left\{x_{i}, \sigma_{i}\right\}\right)=\prod_{i<j}\left|z_{i}-z_{j}\right|^{g_{\sigma_{i} \sigma_{j}}} .
$$

The wavefunction is bosonic; for the fermionic LL, one undoes the Jordan-Wigner transformation which leads to:

$$
\Psi_{F, 0}\left(\left\{x_{i}, \sigma_{i}\right\}\right)=\prod_{i<j}\left|z_{i}-z_{j}\right|^{g_{\sigma_{i} \sigma_{j}}}
$$

$$
\times \prod_{i<j}\left\{\left(\frac{\left(z_{i}-z_{j}\right)}{\left|z_{i}-z_{j}\right|}\right)^{\delta_{\sigma_{i} \sigma_{j}}} \exp i \frac{\pi}{2} \operatorname{sgn}\left(\sigma_{i}-\sigma_{j}\right)\right\} .
$$

(The antisymmetrizing factor consists of two parts, one which ensures that particles of the same species anticommute, and a second part known as a Klein factor which allows antisymmetry for particles of different spin.) Let us redefine the matrix elements of $\widehat{g}$ per:

$$
\begin{aligned}
g_{\sigma \sigma^{\prime}} & =\left(\begin{array}{ll}
\lambda & \mu \\
\mu & \lambda
\end{array}\right) \\
K_{c}^{-1} & =\lambda+\mu \\
K_{s}^{-1} & =\lambda-\mu
\end{aligned}
$$

If we denote the coordinates of particles with spin $\uparrow$ and $\downarrow$ respectively by $u$ and $v$ then the ground state can be rewritten as (for convenience the antisymmetrizing factor is omitted):

$$
\Psi_{0}\left(\left\{u_{i}, v_{i}\right\}\right)=\prod_{i<j}\left|u_{i}-u_{j}\right|^{\lambda} \prod_{i<j}\left|v_{i}-v_{j}\right|^{\lambda} \prod_{i, j}\left|u_{i}-v_{j}\right|^{\mu},
$$

$$
\begin{aligned}
\Psi_{c} & =\left[\prod_{i<j}\left|u_{i}-u_{j}\right|\left|v_{i}-v_{j}\right|\left|u_{i}-v_{j}\right|\right]^{1 / 2 K_{c}} \\
& =\prod_{i<j}\left|z_{i}-z_{j}\right|^{1 / 2 K_{c}} \\
\Psi_{s} & =\left[\prod_{i<j}\left|u_{i}-u_{j}\right|\left|v_{i}-v_{j}\right| /\left|u_{i}-v_{j}\right|\right]^{1 / 2 K_{s}} \\
& =\prod_{i<j}\left|z_{i}-z_{j}\right|^{\sigma_{i} \sigma_{j} / 2 K_{s}} .
\end{aligned}
$$

For the fermionic LL the charge part gets an additional factor $\left(\frac{\left(x_{i}-x_{j}\right)}{\left|x_{i}-x_{j}\right|}\right)^{1 / 2}$ and the spin part, a factor $\left(\frac{\left(x_{i}-x_{j}\right)}{\left|x_{i}-x_{j}\right|}\right)^{\sigma_{i} \sigma_{j} / 2} \exp i \frac{\pi}{2} \operatorname{sgn}\left(\sigma_{i}-\sigma_{j}\right)$. 
These are 1D Laughlin multi-component wavefunctions. In 2D they are known as Halperin wavefunctions which describe multi-component systems of the FQHE50. In that context the $\widehat{g}$ matrix is known as Wen's topological $K$ matrix 28. The main difference between the two matrices is that the the entries of the $K$ matrix are integers while $\widehat{g}$ matrix elements are arbitrary real numbers (only constrained to yield positive and real eigenvalues). The $\widehat{g}$ matrix does not allow for a topological interpretation either since there is no topological quantization as in the FQHE. We will call the $\widehat{g}$ matrix, the charge matrix because it corresponds to the couplings between particles in the plasma analogy (see eq.(3.12) 51 .

\section{Elementary excitations : the holon, the spinon, Laughlin quasiparticles.}

We generalize the approach followed for the spinless LL. There is a decoupling of the dynamics at two levels: chiral separation as well as spin-charge separation. In particular both the charge and the spin hamiltonians $H_{c}$ and $H_{s}$ - display chiral separation: $H_{c}=H_{c+}+H_{c-}$ and $H_{s}=H_{s+}+H_{s-}$ where the four hamiltonians all commute $\left(\left[H_{c / s \pm}, H_{c / s \pm}\right]=0\right)$. The following operators create the exact eigenstates of the relevant chiral hamiltonians:

$$
\begin{gathered}
V_{\tau}^{ \pm}\left(Q_{\tau, \pm}, q\right)=\int d x \exp i q x \exp -i \sqrt{\pi / 2} Q_{\tau, \pm} \Theta_{\tau, \pm} \\
\Theta_{\tau, \pm}=\Theta_{\tau} \mp \Phi_{\tau} / K_{\tau} ; \tau=c, s \\
q=\frac{2 \pi n}{L} \mp \frac{2 \pi}{L} \frac{Q_{\tau, \pm}^{2}}{K_{\tau}} \\
Q_{\tau, \pm}=\frac{Q_{\uparrow}+\tau Q_{\downarrow}}{2} \pm K_{\tau} \frac{J_{\uparrow}+\tau J_{\downarrow}}{2}
\end{gathered}
$$

(The square root $\sqrt{2}$ in the exponential comes from the normalization of the charge and spin fields; $c$ and $s$ index charge and spin respectively; $\tau= \pm 1$ for charge and spin respectively.) One easily checks that:

$$
\left[\widehat{Q}_{\uparrow}+\tau \widehat{Q}_{\downarrow}, V^{ \pm}\left(Q_{\tau^{\prime}, \pm}\right)\right]=\delta_{\tau \tau^{\prime}} Q_{\tau, \pm} V^{ \pm}\left(Q_{\tau^{\prime}, \pm}\right) .
$$

This implies that these excitations either carry a charge $Q=Q_{c, \pm}$ but then have no spin (the operators $V_{c}^{ \pm}$), or that they have a spin $S_{z}=S_{ \pm}=Q_{s, \pm} / 2$ but no charge (operators $V_{s}^{ \pm}$).As expected the fractional states come in two brands: the first corresponds to charge excitations and the second to spin excitations. Hereafter we will note the charge and the spins of these excitations as

$$
\begin{aligned}
Q_{ \pm} & =Q_{c \pm}, \\
S_{ \pm} & =\frac{Q_{s, \pm}}{2} .
\end{aligned}
$$

Because of the obvious relevance to physical systems we focus first on elementary excitations for a fermionic LL; we will consider the case of a bosonic LL later in section (III Q). Solving the constraints on the charge and current which again obey the selection rule $Q_{\uparrow}-J_{\uparrow}=2 n_{\uparrow}$ and $Q_{\downarrow}-J_{\downarrow}=2 n_{\downarrow}$ ( the $n$ are integers), we find:

$$
\begin{aligned}
\left(\begin{array}{l}
Q_{+} \\
Q_{-} \\
S_{+} \\
S_{-}
\end{array}\right)= & n_{\uparrow}\left(\begin{array}{l}
1 \\
1 \\
1 / 2 \\
1 / 2
\end{array}\right)+n_{\downarrow}\left(\begin{array}{l}
1 \\
1 \\
-1 / 2 \\
-1 / 2
\end{array}\right) \\
& +J_{\uparrow}\left(\begin{array}{l}
\frac{\frac{1+K_{c}}{2}}{\frac{1-K_{c}}{2}} \\
\frac{1+K_{s}}{1-K_{s}}
\end{array}\right)+J_{\downarrow}\left(\begin{array}{l}
\frac{1+K_{c}}{1-K_{c}} \\
-\frac{1+K_{s}}{4} \\
-\frac{1-K_{s}}{4}
\end{array}\right)
\end{aligned}
$$

This compact equation must be read as follows. Each entry represents a fractional excitation; the first two lines are charge spinless excitations, while the last two lines represent spin excitations. For instance the entry $Q_{+}$ is associated with a fractional excitation with charge $Q=Q_{+}$which carries no spin, and propagates in the right direction: therefore $\frac{1+K_{c}}{2}$ in the first line means a spinless state with charge $Q=\frac{1+K_{c}}{2}$ going to the right. Likewise the second line characterizes charge excitations propagating to the left. The line $S_{+}$means that the states have no charge, a spin component $S_{z}=S_{+}$ and propagate to the right: for instance $1 / 2$ is a spin one-half fractional state. Each line gives the decomposition of a given fractional excitation into elementary excitations: for instance the $Q_{+}$excitation is made up of $n_{\uparrow}+n_{\downarrow}$ excitations $V_{c}^{+}(Q=1)$, and $J_{\uparrow}+J_{\downarrow}$ excitations $V_{c}^{+}\left(Q=\frac{1+K_{c}}{2}\right)$. The previous equation summarizes the selection rules which are obeyed by the elementary excitations.

Let us give an example. Suppose one adds a spin up electron at the Fermi level in the Luttinger liquid. This is a $Q_{\uparrow}=1=J_{\uparrow}$ and $Q_{\downarrow}=0=J_{\downarrow}$ excitation or in terms of $n_{\uparrow}$ and $n_{\downarrow}$, this is a $\left(n_{\uparrow}=0, n_{\downarrow}=0, J_{\uparrow}=1, J_{\downarrow}=0\right)$ state. Equation (3.29) shows that the spinor

$$
\left(\begin{array}{l}
Q_{+} \\
Q_{-} \\
S_{+} \\
S_{-}
\end{array}\right)=\left(\begin{array}{l}
\frac{1+K_{c}}{2} \\
\frac{1-K_{c}}{2} \\
\frac{1+K_{s}}{4} \\
\frac{1-K_{s}}{4}
\end{array}\right)
$$

is created; this means that the spin up electron added at the Fermi level $k_{F}$ splits into four fractional states: a charge $\frac{1+K_{c}}{2}$ anyon propagating at velocity $u_{c}$; this state has no spin; a second charge anyon with charge $\frac{1-K_{c}}{2}$ and velocity $-u_{c}$ and then two spin anyons with velocities $u_{s}$ and $-u_{s}$ and respective spin $S_{z}=\frac{1 \pm K_{s}}{4}$. In the special case of spin rotational invariance $\left(K_{s}=1\right)$ there is only one spin anyon: the spinon with spin $S_{z}=1 / 2$ which propagates to the right with velocity $u_{s}$ ( or to the left with velocity $-u_{s}$ if the electron had been added at the left Fermi point $-k_{F}$ ). Likewise, if $K_{c}=1$, there is 
a single charge state, which has charge $Q=1$. In the non-interacting case, the charge velocity $u_{c}$ and the spin velocity $u_{s}$ are equal and therefore the spinon and the charge 1 state subsume into a single state since they move in the same direction with the same velocity: we have just recovered the spin up electron.

a. Holon and spinon. Let us identify the content of the elementary excitations, starting with $V_{c}^{ \pm}(Q=1)$ and $V_{s}^{ \pm}\left(S_{z}=1 / 2\right)$. The charge and the spin carried by these fractional excitations make it reasonable to interpret them as the holon and the spinon respectively.

The physical processe involved in the creation of each of these states confirms this identification. Indeed the minimal operation which involves $V_{c}^{ \pm}(Q=1)$ is obtained when $J_{\uparrow}=0=J_{\downarrow}$ and set $n_{\uparrow}=1=n_{\downarrow}$ in equation (3.29). This is an excitation for which $Q_{\uparrow}=1=Q_{\downarrow}$ and $J_{\uparrow}=$ $0=J_{\downarrow}$ which means that this is a pure charge process (no spin variation $S_{z}=\left(Q_{\uparrow}-Q_{\downarrow}\right) / 2=0$, no spin current nor charge current). $V_{c}^{ \pm}(Q=1)$ is an excitation associated with the addition of charge in the LL. All the transitions in Fock space which occur after adding a charge to the ground state therefore involve $V_{c}^{ \pm}(Q=1)$. If is then consistent to identify $V_{c}^{ \pm}(Q=1)$ as the holon 52 .

Likewise the minimal excitation generating $V_{s}^{ \pm}\left(S_{z}=\right.$ $1 / 2)$ is a spin one transition which is a pure spin process. All excitations for which there is a spin flip will therefore create $V_{s}^{ \pm}\left(S_{z}=1 / 2\right)$ (in pairs). This is what we expect from a spinon.

Notice that both for the holon and spinon there are even-odd effects arising in the low-energy gaussian theory. Indeed, equation (3.29) shows that an excitation with includes a spin one-half transition will not create a spinon (we have instead a "hybrid" spin excitation): one needs at least a spin one transition to crate a spinon. This means that adding a single electron does not create a spinon: even number of electrons are required. This makes sense since for a spin chain the minimal spin excitation is also a spin-flip which involves two electrons and not just one. The same behaviour is observed with the holon: the minimal process which creates it adds two electrons $\left(Q=2\right.$ since $\left.Q_{\uparrow}=1=Q_{\downarrow}\right)$. These even-odd effects are a direct consequence of statistics and would not be observed with a bosonic two component LL.

With eq.(3.11), we find that the wavefunctions for the holon and the spinon are simply (with $z=\exp i \frac{2 \pi}{L} x$ ):

$$
\begin{aligned}
& \Psi_{\text {holon }}\left(z_{0}\right)=V_{c}^{+}\left(Q_{c}=1, z_{0}\right) \Psi_{c} \\
& =\prod_{i}\left(\frac{\left(x_{i}-x_{0}\right)}{\left|x_{i}-x_{0}\right|}\right)^{\frac{1}{2 K_{c}}} \prod_{i}\left|z_{i}-z_{0}\right|^{1 / 2 K_{c}} \\
& \times \prod_{i<j}\left|z_{i}-z_{j}\right|^{1 / 2 K_{c}} \prod_{x_{0}, i<j}\left\{\frac{\left(x_{i}-x_{j}\right)}{\left|x_{i}-x_{j}\right|}\right\}^{1 / 2} \\
& =\prod_{i}\left(z_{i}-z_{0}\right)^{1 / 2 K_{c}} \prod_{i<j}\left|z_{i}-z_{j}\right|^{1 / 2 K_{c}}
\end{aligned}
$$

and:

$$
\begin{aligned}
& \Psi_{\text {spinon }}\left(\sigma_{0}, z_{0}\right) \\
= & V_{s}^{+}\left(S_{z}=\sigma_{0} / 2, z_{0}\right) \Psi_{s} \\
= & \prod_{i}\left(\frac{\left(x_{i}-x_{0}\right)}{\left|x_{i}-x_{0}\right|}\right)^{\sigma_{0} \sigma_{i} / 2 K_{s}} \prod_{i}\left|z_{i}-z_{0}\right|^{\sigma_{0} \sigma_{i} / 2 K_{s}} \\
& \times \prod_{i<j}\left|z_{i}-z_{j}\right|^{\sigma_{i} \sigma_{j} / 2 K_{s}} \prod_{x_{0}, i<j}\left\{\frac{\left(x_{i}-x_{j}\right)}{\left|x_{i}-x_{j}\right|}\right\}^{\sigma_{i} \sigma_{j} / 2} .
\end{aligned}
$$

The holon and the spinon are both anyons with exchange statistics:

$$
\begin{aligned}
& \theta_{c}=\frac{\pi}{2 K_{c}} \\
& \theta_{s}=\frac{\pi}{2 K_{s}}
\end{aligned}
$$

(the statistics were computed in subsection [B]). Except for the special case $K_{\tau}=1$, these objects are not semions; in addition, for holons we must also require $v_{c} \neq v_{s}$ to ensure spin-charge separation. Contrast our results (eqns (3.31) and (3.33)) with the commonly used but incorrect charge-spin decoupling of the electron operator 5 . The holon and the spinon generalize the dual excitation found for the spinless LL; in the same way, duality transforms the holon and the spinon into the two-component generalizations of the Laughlin quasiparticles. This is a most remarkable yet simple result because it shows that two seemingly unrelated fractional excitations -the holon (or spinon) and the Laughlin quasiparticle- occurring in two very different contexts are actually deeply connected.

Similarly to the spinless LL, in addition to the holon and to the spinon, we hybrid excitations complete the basis of fractional excitations. Their charge and spin are intermediate between those of the holon and spinon and those of their dual excitations, the Laughlin quasiparticles which we discuss now.

b. Laughlin quasiparticles. We can choose another basis of elementary excitations dual to the previous one which will parametrize the excitations in terms of current processes and electron addition at the Fermi surface. This basis, emphasizing Laughlin quasiparticles as elementary excitations reads:

$$
\begin{aligned}
\left(\begin{array}{l}
Q_{+} \\
Q_{-} \\
S_{+} \\
S_{-}
\end{array}\right)= & n_{\uparrow}\left(\begin{array}{l}
K_{c} \\
-K_{c} \\
K_{s} / 2 \\
-K_{s} / 2
\end{array}\right)+n_{\downarrow}\left(\begin{array}{l}
K_{c} \\
-K_{c} \\
-K_{s} / 2 \\
K_{s} / 2
\end{array}\right) \\
& +Q_{\uparrow}\left(\begin{array}{l}
\frac{1+K_{c}}{\frac{1-K_{c}}{2}} \\
\frac{1+K_{s}}{4} \\
\frac{1-K_{s}}{4}
\end{array}\right)+Q_{\downarrow}\left(\begin{array}{l}
\frac{1+K_{c}}{2} \\
\frac{1-K_{c}}{2} \\
-\frac{1+K_{s}}{4} \\
-\frac{1-K_{s}}{4}
\end{array}\right),
\end{aligned}
$$


where again $Q_{\uparrow}-J_{\uparrow}=2 n_{\uparrow}$ and $Q_{\downarrow}-J_{\downarrow}=2 n_{\downarrow}$. In addition to the hybrid quasiparticles $V_{c}^{ \pm}\left(Q=\frac{1 \pm K_{c}}{2}\right)$ and $V_{s}^{ \pm}\left(S_{z}=\frac{1 \pm K_{s}}{4}\right)$ which already existed in the previous basis, we have two excitations associated with pure charge current or spin current processes: $V_{c}\left(Q_{c}=K_{c}\right)$ and $V_{s}\left(S_{z}=K_{s} / 2\right)$. Under electromagnetic duality the latter are conjugate to the holon and spinon respectively. Actually they are obtained by spin-charge separation of the two-component Laughlin quasiparticle, and we may call them a Laughlin holon and a Laughlin spinon. Let us compute the wavefunctions of these two excitations; one finds:

$$
\begin{gathered}
V_{c}\left(Q_{c}=K_{c}, z_{0}\right) \Psi_{c} \\
=\prod_{i}\left(z_{i}-z_{0}\right)^{1 / 2} \\
\times \exp -i 2 k_{F}\left(\frac{\sum x_{i}}{N}+x_{0}\right) \Psi_{c}, \\
V_{s}\left(S^{z}=\sigma_{0} K_{s} / 2, z_{0}\right) \Psi_{s} \\
=\prod_{i}\left(z_{i}-z_{0}\right)^{\sigma_{i} \sigma_{0} / 2} \\
\times \exp -i 2 \sigma_{0}\left(k_{\uparrow}-k_{\downarrow}\right)\left(\frac{\sum x_{i \uparrow}-x_{j \downarrow}}{M}+x_{0}\right) \Psi_{s}
\end{gathered}
$$

In the previous expression $\sigma_{0}$ takes on the values \pm 1 and $k_{\uparrow}, k_{\downarrow}$ are the Fermi vectors associated with particles of spin up and down: $k_{\sigma}=\frac{\pi}{L} N_{\sigma}$ and $N=N_{\uparrow}-N_{\downarrow}$, $M=N_{\uparrow}-N_{\downarrow} \cdot \Psi_{c}$ and $\Psi_{s}$ are given for the bosonic LL in eq.(3.20) and (3.21); for the fermionic LL, there are additional phase factors given in the text following eq.(3.20) and (3.21). Plasma analogy allows to get the charge and spin of the Laughlin holon eq.(3.37) and spinon eq.(3.39)). The Laughlin holon and spinon are the stable excitations into which the Laughlin quasiparticle decays as a result of spin-charge separation; in the localized Wannier basis we have considered throughout, the product of the wavefunctions of the two excitations yields indeed:

$$
\Psi_{q p}\left(z_{0}, \sigma_{0}\right)=\prod_{i}\left(z_{i}-z_{0}\right)^{\delta_{\sigma_{0} \sigma_{i}}} \prod_{i<j}\left|z_{i}-z_{j}\right|^{g_{\sigma_{i} \sigma_{j}}},
$$

which is just the generalization of the Laughlin quasiparticle to two component systems: when we add spin, the Laughlin quasiparticle comes in two flavours (up or down) and the Laughlin correlation hole acts only on particles of the same flavour. It therefore carries both fractional charge and fractional spin. (It would be spinless if the Laughlin prefactor were $\prod_{i}\left(z_{i}-z_{0}\right)$ instead of $\left.\prod_{i}\left(z_{i}-z_{0}\right)^{\delta_{\sigma_{0} \sigma_{i}}}\right)$.

The Laughlin holon and spinon have statistical phases $\theta_{c}=\pi K_{c}$ and $\theta_{s}=\pi K_{s} / 2$. For a spin-rotational invariant system, the Laughlin spinon and its dual conjugate the spinon - are identical states $\left(K_{s}=1\right.$ is the self-dual point for spin excitations).

\section{B. Luttinger liquid without spin-charge separation.}

\section{The general LL and the charge matrix.}

We now generalize the standard LL theory to include situations with no spin-charge separation. We start from the ground state of the gaussian hamiltonian:

$$
\Psi_{0}[\widehat{g}]=\exp \frac{1}{2} \iint d x d x^{\prime} \rho_{\sigma}(x) g_{\sigma \sigma^{\prime}} \ln \left|\sin \frac{\pi\left(x-x^{\prime}\right)}{L}\right| \rho_{\sigma^{\prime}}\left(x^{\prime}\right),
$$

$$
\begin{aligned}
g_{\sigma \sigma^{\prime}} & =\left(\begin{array}{ll}
\lambda & \mu \\
\mu & \lambda
\end{array}\right), \\
K_{c}^{-1} & =\lambda+\mu, \\
K_{s}^{-1} & =\lambda-\mu .
\end{aligned}
$$

and relax the constraint $g_{\uparrow \uparrow}=g_{\downarrow \downarrow}$ (while $\widehat{g}$ is kept symmetric). We consider the charge matrix: $g_{\sigma \sigma^{\prime}}=\left(\begin{array}{ll}\lambda & \mu \\ \mu & \lambda^{\prime}\end{array}\right)$ and the associated wavefunction $\Psi_{0}[\widehat{g}]$. We introduce for convenience the eigenvalues of the charge matrix and the unitary matrix $P$ :

$$
\begin{aligned}
P^{-1} \widehat{g} P & =\widehat{D}=\left(\begin{array}{ll}
1 / K_{1} & 0 \\
0 & 1 / K_{2}
\end{array}\right) \\
P_{\sigma \tau} P_{\sigma^{\prime} \tau^{\prime}} g_{\sigma \sigma^{\prime}} & =\frac{\delta_{\tau \tau^{\prime}}}{K_{\tau}} ; \quad \tau=1,2
\end{aligned}
$$

The normal modes of the charge matrix are simply:

$$
\begin{gathered}
\rho_{\tau}=P_{\sigma \tau} \rho_{\sigma} \Leftrightarrow \rho_{\sigma}=P_{\sigma \tau} \rho_{\tau} \tau=1,2 \\
\sum_{\sigma \sigma^{\prime}} \rho_{\sigma}(x) g_{\sigma \sigma^{\prime}} \rho_{\sigma^{\prime}}\left(x^{\prime}\right)=\sum_{\tau} \rho_{\tau}(x) \frac{1}{K_{\tau}} \rho_{\tau}\left(x^{\prime}\right) .
\end{gathered}
$$

If the charge matrix obeys a $Z_{2}$ symmetry then the normal modes are just the charge and spin density (up to a normalization factor) $\rho_{1}=\rho_{c} / \sqrt{2}$ and $\left.\rho_{2}=\rho_{s} / \sqrt{2}\right)$. The wavefunction now reads:

$$
\begin{aligned}
& \Psi_{0}[\widehat{g}] \\
= & \exp \frac{1}{2 K_{1}} \iint d x d x^{\prime} \rho_{1}(x) \ln \left|\sin \frac{\pi\left(x-x^{\prime}\right)}{L}\right| \rho_{1}\left(x^{\prime}\right) \\
\times & \exp \frac{1}{2 K_{2}} \iint d x d x^{\prime} \rho_{2}(x) \ln \left|\sin \frac{\pi\left(x-x^{\prime}\right)}{L}\right| \rho_{2}\left(x^{\prime}\right) .
\end{aligned}
$$

We have expressed the ground state in this decoupled form because this allows us to directly write down a gaussian hamiltonian with ground state $\Psi_{0}[\widehat{g}]$. This generalizes the spin-charge decoupled gaussian theory. We introduce the phase fields associated with the normal densities $\rho_{1}$ and $\rho_{2}$ 


$$
\begin{gathered}
\rho_{\tau}=-\frac{1}{\sqrt{\pi}} \partial_{x} \Phi_{\tau}, j_{\tau}=\frac{1}{\sqrt{\pi}} \partial_{x} \Theta_{\tau}, \\
{\left[\Phi_{\tau}(x), \partial_{x} \Theta_{\tau^{\prime}}(y)\right]=i \delta_{\tau \tau^{\prime}} \delta(x-y) .}
\end{gathered}
$$

It is then clear that $\Psi_{0}[\widehat{g}]$ is the exact ground state of the following family of two component gaussian hamiltonians for arbitrary velocities $u_{1}, u_{2}$ :

$$
\begin{aligned}
& H\left[\widehat{g}, u_{1}, u_{2}\right] \\
= & H_{B}\left[u_{1}, K_{1}\right]+H_{B}\left[u_{2}, K_{2}\right] \\
= & \sum_{\tau=1,2} \frac{u_{\tau}}{2} \int_{0}^{L} d x\left[K_{\tau}^{-1}\left(\partial_{x} \Phi_{\tau}\right)^{2}+K_{\tau}\left(\partial_{x} \Theta_{\tau}\right)^{2}\right] .
\end{aligned}
$$

The two hamiltonians $H_{B}\left[u_{1}, K_{1}\right]$ and $H_{B}\left[u_{2}, K_{2}\right]$ commute by construction. The next section will be devoted to the properties of that generalized LL theory. Let us stress here the main property of this general LL now: by construction that theory corresponds to a generalized separation: the normal modes will not be charge and spin modes but mix charge and spin in a proportion fixed in time. This will translate for the fractional excitations to states with both fractional charge and fractional spin.

\section{Main properties.}

The compressibility and spin susceptibility are easily computed and one finds:

$$
\begin{gathered}
\kappa^{-1}=\frac{1}{L} \frac{\partial^{2} E_{0}}{\partial \rho_{0}^{2}}=\frac{\pi}{4} \sum_{\tau} \frac{u_{\tau}}{K_{\tau}}\left(\sum_{\sigma} P_{\sigma \tau}\right)^{2}, \\
\chi_{s}^{-1}=\frac{1}{L} \frac{\partial^{2} E_{0}}{\partial \rho_{s}^{2}}=\frac{\pi}{4} \sum_{\tau} \frac{u_{\tau}}{K_{\tau}}\left(\sum_{\sigma} \sigma P_{\sigma \tau}\right)^{2} .
\end{gathered}
$$

$\rho_{0}$ and $\rho_{s}$ are the charge and spin mean densities, while $E_{0}$ is the ground state energy. The Drude peak is:

$$
D=\frac{1}{L} \frac{\partial^{2} E_{0}}{\partial \phi^{2}}=\sum_{\tau} u_{\tau} K_{\tau}\left(\sum_{\sigma} P_{\sigma \tau}\right)^{2},
$$

where $\phi$ is a flux threading the LL ring of length $L$. This expression can also be recovered using the Kubo formula; one then needs the expression of the current density which one finds with the continuity equation. The current is renormalized as for the spinless LL and the LL with spin-charge separation. In contrast to the case of the LL with spin-charge separation, here the expression involves both $K_{1}$ and $K_{2}$ because both modes one and two involve charge:

$$
j_{R}(x)=\sum_{\sigma}\left(\sum_{\sigma^{\prime}, \tau} u_{\tau} K_{\tau} P_{\sigma \tau} P_{\sigma^{\prime} \tau}\right) \frac{\partial_{x} \Theta_{\sigma}}{\sqrt{\pi}} .
$$

Anomalous exponents are easily computed as functions of the charge matrix $\widehat{g}$ which leads to compact expressions valid both for the LL with spin-charge separation or for the more general LL; one introduces the Fermi vectors: $k_{F \sigma}=\frac{\pi N_{\sigma}}{L}$. For instance density-density correlators are obtained with $H[\widehat{g}]$ and with the bosonization formulas, yielding the static structure factor; the dominant Fourier components are $k=0, k=2 k_{F \uparrow}, k=2 k_{F \downarrow}$ and $k=$ $2 k_{F \uparrow}+2 k_{F \downarrow}$. Near $k=0$ :

$$
\begin{aligned}
& <\delta \rho_{\sigma}(0) \delta \rho_{\sigma^{\prime}}(x)>_{k=0}=\frac{\widehat{g^{-1}} \sigma \sigma^{\prime}}{2(\pi x)^{2}}, \\
& <\delta \rho(0) \delta \rho(x)>=\frac{A_{k=0}}{(\pi x)^{2}} ; A_{k=0}=\sum_{\sigma \sigma^{\prime}} \frac{\widehat{g^{-1}} \sigma \sigma^{\prime}}{2} .
\end{aligned}
$$

For the higher harmonics one includes a mode at $2 k_{F \uparrow}+2 k_{F \downarrow}=2 \pi \rho$ (which appears in the Hubbard model in a magnetic field):

$$
\begin{aligned}
& <\delta \rho(0) \delta \rho(x)>=\frac{A_{k=0}}{(\pi x)^{2}}+a_{\uparrow} \frac{\cos \left(2 k_{F \uparrow} x\right)}{x^{2+\alpha\left(2 k_{F \uparrow}\right)}}+a_{\downarrow} \frac{\cos 2 k_{F \downarrow} x}{x^{2+\alpha\left(2 k_{F \downarrow}\right)}} \\
& +b \frac{\cos \left(2 k_{F \uparrow}+2 k_{F \downarrow}\right) x}{x^{2+\alpha\left(2 k_{F \uparrow}+2 k_{F \downarrow}\right)}}
\end{aligned}
$$

$A_{k=0}$ is fixed in the low-energy limit but the other constants $a_{\uparrow}, a_{\downarrow}, b$ are non-universal and depend on highenergy processes. When there is spin-charge separation, the exponent for $4 k_{F}$ oscillations and the constant $A_{k=0}$ are related by the equation $2+\alpha\left(4 k_{F}\right)=4 A_{k=0}$; in the general case, we find:

$$
2+\alpha\left(2 k_{F \uparrow}+2 k_{F \downarrow}\right)=4 A_{k=0}
$$

The derivation of the exponents is done in exactly the same manner as in the spin-charge separated LL.

Spin-spin correlation functions are:

$$
\begin{gathered}
<S_{z}(0) S_{z}(x)>=\frac{\sum_{\sigma \sigma^{\prime}}\left(\sigma \sigma^{\prime} \widehat{g^{-1}} \sigma \sigma^{\prime}\right)}{2(\pi x)^{2}} \\
+\sum_{\sigma} \frac{\cos 2 k_{\sigma} x}{|x|^{2 \widehat{g}_{\sigma \sigma}^{-1}}}, \\
<S^{+}(0) S^{-}(x)>=\frac{\cos \left(k_{F \uparrow}+k_{F \downarrow}\right) x}{|x|^{\gamma}} \\
\gamma=\left[g_{\uparrow \downarrow}^{-1}-g_{\uparrow \downarrow}+\frac{1}{2} \sum_{\sigma}\left(\widehat{g}^{-1}+\widehat{g}\right)_{\sigma \sigma}\right] .
\end{gathered}
$$

Electronic Green functions decay as: 


$$
<\Psi_{\sigma}(0) \Psi_{\sigma}^{+}(x)>=\frac{\exp i k_{\sigma} x}{|x|^{1+\alpha(\sigma)}} ; 1+\alpha_{F}(\sigma)=\frac{1}{2}\left(\widehat{g}+\widehat{g}^{-1}\right)_{\sigma \sigma} .
$$

For bosons the exponent is modified as: $1+\alpha_{B}(\sigma)=$ $\frac{1}{2} \widehat{g}_{\sigma \sigma}$. These exponents are derived with the bosonization formulas but can also be found by plasma analogy.

\section{The charge matrix: a summary.}

For the two-component LL there are three interesting situations which we summarize below:

i) in the general case, the (symmetric) $\widehat{g}$ matrix has arbitrary entries; there is no spin-charge separation but a more general two-mode separation:

$$
\widehat{g}=\left(\begin{array}{cc}
\lambda & \mu \\
\mu & \lambda^{\prime}
\end{array}\right) .
$$

As will be shown below, the Hubbard model in a magnetic field can be described by such a theory.

ii) the $\widehat{g}$ matrix has a $Z_{2}$ symmetry; this case pertains to spin-charge separation:

$$
\widehat{g}=\left(\begin{array}{cc}
\lambda & \mu \\
\mu & \lambda
\end{array}\right), K_{\rho}=\frac{1}{\lambda+\mu}, K_{\sigma}=\frac{1}{\lambda-\mu}
$$

Indeed the symmetry under the exchange of up and down spins implies that the normal modes of the charge matrix are just the charge and spin modes. The LL parameters are then the eigenvalues of the inverse of the charge matrix. This situation describes models with spin-charge separation but with a spin anisotropy, for instance a Hubbard model to which one would add some Ising term $\sum_{n} S_{z}(n) S_{z}(n+1)$.

iii) the $\widehat{g}$ matrix corresponds to a $S U(2)$ symmetric case $\left(K_{\sigma}=1\right)$ :

$$
\widehat{g}=\left(\begin{array}{ll}
\mu+1 & \mu \\
\mu & \mu+1
\end{array}\right), \quad K_{\rho}=\frac{1}{2 \mu+1}, K_{\sigma}=1
$$

This situation describes the low-energy limit of the Hubbard model. It is noteworthy that the wavefunctions $\Psi[\widehat{g}]$ for that sub-case were used in a variational approach of the 1D $t-J$ model giving very good results although it was not realized they were the exact ground states of the gaussian model 54 . The reason why it is so is now transparent.

\section{Elementary excitations for the generalized LL.}

We now consider the excitations of the general bosonic and fermionic LL with or without spin-charge separation. Let us inject particles in the Luttinger liquid. In real space this is described by the operator:

$$
V(x)=\exp -i \sqrt{\pi} \sum_{\sigma}\left(Q_{\sigma} \Theta_{\sigma}(x)-J_{\sigma} \Phi_{\sigma}(x)\right) .
$$

Fractionalization stems from two decouplings: chiral separation and a separation for the internal quantum number generalizing spin-charge separation. In terms of the normal modes fields $\Theta_{\tau}$ and $\Phi_{\tau}(\tau=1,2)$ :

$$
V=\exp -i \sqrt{\pi} \sum_{\tau}\left(\left(\sum_{\sigma} P_{\sigma \tau} Q_{\sigma}\right) \Theta_{\tau}-\left(\sum_{\sigma} P_{\sigma \tau} J_{\sigma}\right) \Phi_{\tau}\right) .
$$

The chiral fields are:

$$
\Theta_{\tau, \pm}(x)=\Theta_{\tau}(x) \mp \Phi_{\tau}(x) / K_{\tau},
$$

and therefore:

$$
V(x)=\prod_{\tau, \pm} \exp -i \sqrt{\pi} Q_{\tau, \pm} \Theta_{\tau, \pm}(x) .
$$

This expression explicitly shows a decoupling into four components. We have defined in the above the chiral charges:

$$
Q_{\tau, \pm}=\frac{1}{2}\left[\left(\sum_{\sigma} P_{\sigma \tau} Q_{\sigma}\right) \pm K_{\tau}\left(\sum_{\sigma} P_{\sigma \tau} J_{\sigma}\right)\right] .
$$

The following operators are exact eigenstates of each chiral hamiltonian $H_{ \pm, \tau} \tau=1,2$ :

$$
\begin{aligned}
V_{\tau}^{ \pm}\left(Q_{\tau, \pm}, q\right) & =\int d x \exp i q x \exp -i \sqrt{\pi} Q_{\tau, \pm} \Theta_{\tau, \pm}(x), \\
q & =\frac{2 \pi n}{L} \mp \frac{2 \pi}{L} \frac{Q_{\tau, \pm}^{2}}{K_{\tau}} .
\end{aligned}
$$

The chiral charges correspond to the charge and spin carried by each of these excitations up to a normalization factor:

$$
\left[\widehat{Q}_{\sigma}, V_{\tau}^{ \pm}\left(Q_{\tau, \pm}, q\right)\right]=Q_{\tau, \pm} P_{\sigma \tau} V_{\tau}^{ \pm}\left(Q_{\tau, \pm}, q\right)
$$

which implies that the charge and spin of $V_{\tau}^{ \pm}\left(Q_{\tau, \pm}, q\right)$ are:

$$
\begin{aligned}
Q & =Q_{\tau, \pm}\left(\sum_{\sigma} P_{\sigma \tau}\right), \\
S_{z} & =Q_{\tau, \pm}\left(\frac{1}{2} \sum_{\sigma} \sigma P_{\sigma \tau}\right) .
\end{aligned}
$$

Thus for an arbitrary charge matrix, fractional excitations carry both charge and spin. However the ratio of charge to spin is constant for each given mode $\tau=1,2$ : $\left(\frac{1}{2} \sum_{\sigma} \sigma P_{\sigma \tau}\right) Q=S_{z}\left(\sum_{\sigma} P_{\sigma \tau}\right)$; of course the phonons associated to each mode mix charge and spin in exactly the same proportions since: 


$$
\begin{aligned}
\rho_{\tau}(x) & =\left(\frac{1}{2} \sum_{\sigma} P_{\sigma \tau}\right) \rho_{c}(x)+\left(\frac{1}{2} \sum_{\sigma} \sigma P_{\sigma \tau}\right) \rho_{s}(x) \\
& =\left(\frac{1}{2} \sum_{\sigma} P_{\sigma \tau}\right) \rho_{c}(x)+\left(\sum_{\sigma} P_{\sigma \tau}\right) s_{z}(x)
\end{aligned}
$$

where $s_{z}(x)$ is a spin density.

It is convenient to define the charge to spin ratio:

$$
r=\frac{Q}{2 S_{z}}
$$

for each mode. Unitary implies that if for the first mode:

$$
r=\frac{Q}{2 S_{z}}=p
$$

then for the second mode:

$$
r=\frac{Q}{2 S_{z}}=-\frac{1}{p} .
$$

where $p$ is arbitrary. Note that for a Fermi liquid these ratios are $r= \pm 1$ (we are characterizing Landau quasiparticles (or holes) of either spin) and when spin-charge separation is realized the ratio is either $r=0$ or $r= \pm \infty$.

Let us give the elementary excitations. The simplest case is that of bosons:

$$
\begin{gathered}
Q_{\tau, \pm}=Q_{\uparrow}\left(\frac{P_{\uparrow \tau}}{2}\right)+Q_{\downarrow}\left(\frac{P_{\downarrow \tau}}{2}\right) \\
+\frac{J_{\uparrow}}{2}\left( \pm P_{\uparrow \tau} K_{\tau}\right)+\frac{J_{\downarrow}}{2}\left( \pm P_{\downarrow \tau} K_{\tau}\right) .
\end{gathered}
$$

To simplify the notation, we have only written a single line, but $Q_{\tau, \pm}$ and the other entries should be read as four-vectors. $Q_{\sigma}$ and $\frac{J_{\sigma}}{2}$ are arbitrary independent integers, which shows that the states $V_{\tau}^{ \pm}\left(\widetilde{Q}_{\tau, \pm}, q\right)$ where $\widetilde{Q}_{\tau, \pm}=\frac{P_{\uparrow \tau}}{2}, \frac{P_{\downarrow \tau}}{2}, \pm P_{\uparrow \tau} K_{\tau}$ or $\pm P_{\downarrow \tau} K_{\tau}$ are elementary excitations. As an illustration let us consider the simple case of a $Z_{2}$ symmetric charge matrix for bosons which have a pseudo-spin index. The unitary matrix $P$ is:

$$
P_{\sigma \tau}=\left(\begin{array}{ll}
1 / \sqrt{2} & 1 / \sqrt{2} \\
1 / \sqrt{2} & -1 / \sqrt{2}
\end{array}\right)
$$

Then it follows from eq.(3.77) and (3.78) that for mode $\tau=1$ (the charge mode) $Q=\sqrt{2} Q_{\tau, \pm}$ and $S_{z}=0$; for mode $\tau=2 Q=0$ but $S_{z}=Q_{\tau, \pm} / \sqrt{2}$. If we take these normalizations into account:

$$
\begin{aligned}
\left(\begin{array}{l}
Q_{+} \\
Q_{-} \\
S_{+} \\
S_{-}
\end{array}\right)= & Q_{\uparrow}\left(\begin{array}{l}
1 / 2 \\
1 / 2 \\
1 / 4 \\
1 / 4
\end{array}\right)+Q_{\downarrow}\left(\begin{array}{l}
1 / 2 \\
1 / 2 \\
-1 / 4 \\
-1 / 4
\end{array}\right) \\
+ & \frac{J_{\uparrow}}{2}\left(\begin{array}{l}
K_{c} \\
-K_{c} \\
K_{s} / 2 \\
-K_{s} / 2
\end{array}\right)+\frac{J_{\downarrow}}{2}\left(\begin{array}{l}
K_{c} \\
-K_{c} \\
-K_{s} / 2 \\
K_{s} / 2
\end{array}\right)
\end{aligned}
$$

Once again we find a charge $1 / 2$ particle and a charge $K_{c}$ Laughlin quasiparticle as for the bosonic spinless LL. But in addition we find new states resulting from a fractionalization of "pseudo-spin" for the bosons (halfspinons for instance).

For the fermionic LL the elementary excitations are obtained by the equation:

$$
\begin{aligned}
Q_{\tau, \pm}= & Q_{\uparrow}\left(P_{\uparrow \tau} \frac{1 \pm K_{\tau}}{2}\right)+Q_{\downarrow}\left(P_{\downarrow \tau} \frac{1 \pm K_{\tau}}{2}\right) \\
& +n_{\uparrow}\left(\mp P_{\uparrow \tau} K_{\tau}\right)+n_{\downarrow}\left(\mp P_{\downarrow \tau} K_{\tau}\right),
\end{aligned}
$$

where we have resolved the constraint: $Q_{\sigma}-J_{\sigma}=2 n_{\sigma}$. This fully characterizes the low-energy elementary excitations of a LL in a magnetic field (see below).

\section{Application to the Hubbard model.}

To illustrate the previous results we discuss the Hubbard model in one dimension. The model was solved exactly by Bethe Ansatz by Lieb and $\mathrm{Wu}$. In zero magnetic field, for repulsive $(U>0)$ interactions, a LL metallic phase exists both for weak and strong coupling, except at half-filling. For very large $U$ the spin-charge decoupling is valid at all energy scales. This was shown by Ogata and Shiba who also found that the Bethe Ansatz ground state then took a remarkable factorized form 55 : it is the product of a charge part (a Slater determinant for free fermions involving all electrons ) and a Bethe wavefunction similar to that of the Heisenberg model on a reduced lattice from which one has removed the holes

$$
\begin{aligned}
& \Psi_{\text {Hubbard }}\left(x_{i}, \sigma_{i}\right) \\
= & \operatorname{det}\left(\exp i k_{j} r_{i},\left|k_{j}\right| \leq k_{F}\right) \Psi_{\text {Heisenberg }}\left(y_{i}, \sigma_{i}\right)
\end{aligned}
$$

$\left(y_{i}\right.$ is the coordinate in the reduced lattice of particle $i$ whose real position is $x_{i}$ ).

It is instructive to compare it to the two-component Jastrow wavefunctions which are also explicitly spincharge decoupled. The Slater determinant is rewritten as (in terms of the circular coordinates $z$ ):

$$
\Psi_{\text {Hubbard }}\left(x_{i}, \sigma_{i}\right)=\prod_{i<j}\left(z_{i}-z_{j}\right) \Psi_{\text {Heisenberg }}\left(y_{i}, \sigma_{i}\right)
$$

This is to be compared with:

$$
\begin{aligned}
\Psi= & \prod_{i<j}\left|z_{i}-z_{j}\right|^{1 / 2 K_{c}} \prod_{i<j}\left|z_{i}-z_{j}\right|^{\sigma_{i} \sigma_{j} / 2 K_{s}} \\
& \prod_{i<j}\left\{\left(\frac{\left(z_{i}-z_{j}\right)}{\left|z_{i}-z_{j}\right|}\right)^{\delta_{\sigma_{i} \sigma_{j}}} \exp i \frac{\pi}{2} \operatorname{sgn}\left(\sigma_{i}-\sigma_{j}\right)\right\}
\end{aligned}
$$

Or if we separate spin and charge: 


$$
\begin{aligned}
\Psi_{c}= & \prod_{i<j}\left[\left|z_{i}-z_{j}\right|^{1 / 2 K_{c}}\left(\frac{\left(z_{i}-z_{j}\right)}{\left|z_{i}-z_{j}\right|}\right)^{1 / 2}\right], \\
\Psi_{s}= & \prod_{i<j}\left[\left|z_{i}-z_{j}\right|^{\sigma_{i} \sigma_{j} / 2 K_{s}}\right. \\
& \left.\times\left(\frac{\left(z_{i}-z_{j}\right)}{\left|z_{i}-z_{j}\right|}\right)^{\sigma_{i} \sigma_{j} / 2} \exp i \frac{\pi}{2} \operatorname{sgn}\left(\sigma_{i}-\sigma_{j}\right)\right] .
\end{aligned}
$$

The spin part of the Laughlin ground state is just the Haldane-Shastry wavefunction if $K_{s}=1$ (rotational invariance) which has the same large-distance physics as the Heisenberg ground state. We can also determine $K_{c}$ without any computation by just reading off its value from the wavefunctions: the charge parts of the two wavefunctions coincide if $K_{c}=1 / 2$ which indeed is the known value of the LL parameter for large $U$.

Bethe Ansatz gives the spectrum and the eigenstates; however it is very difficult to compute correlation functions. An important advance came however with the works of Frahm and Korepin who used CFT in conjunction with Bethe Ansatz to compute critical exponents25. If a theory is conformally invariant, one can show that the finite-size energies of excitations are directly related to their operator dimension (which is one-half of the anomalous dimension of their correlation function). By using Woynarovich's Bethe Ansatz calculations for the finitesize spectrum to order $1 / L$ which he computed within a so-called "dressed charge matrix formalism" 56, Frahm and Korepin were able to extract critical exponents for the correlation functions of the Hubbard model. In particular they found that in the presence of a magnetic field, spin-charge separation was not realized. Penc and Solyom later showed that in $1 / L$ the same spectrum derived by Woynarovich could be expressed in terms of a generalized Tomonaga-Luttinger model with interactions described in the g-ology framework; using equation of motion methods they also derived the anomalous exponents 5 . These two approaches give little insight into the nature of the elementary excitations: how are the holon and spinon modified as a function of microscopic parameters? The description of spin-charge separation (or its absence) is not transparent either: the dressed charge matrix tell us little about spin-charge separation; its changes are not easy to relate to that property. This is to be contrasted with our charge matrix formalism in which spin-charge separation is directly connected to a symmetry of the charge matrix $\widehat{g}\left(Z_{2}\right.$ symmetry). We will show that the "dressed charge matrix" of Bethe Ansatz and the charge matrix $\widehat{g}$ are in fact related: the inverse of the symmetric charge matrix is roughly the square of the $Z$ matrix. We will proceed in the following manner: we will show that Woynarovich's finite-size spectrum is identical to that of our generalized LL. This yields the charge matrix $\widehat{g}$ in terms of the dressed charge matrix $Z$ and gives us both the anomalous exponents and the fractional excitations since we already derived them for the generalized LL. The relation of our charge matrix formal- ism to Penc and Solyom g-ology approach is the following: it can be understood as a bosonization of their generalized Tomonaga-Luttinger model; it is much simpler however to work directly within the gaussian hamiltonian framework. Our approach has several advantages in addition to making an explicit contact with the seemingly unrelated physics of Laughlin states: (a) we avoid an ambiguity in the determination of anomalous exponents in Frahm's and Korepin approach;58 (b), we can give the nature of elementary excitations ( and show that they are fractional states in the first place ) and (c) we are able to give a clear criterion of spin-charge separation.

Woynarovich's finite-size spectrum in Frahm and Korepin's notations is the following:

$$
\begin{aligned}
& E\left(\Delta N, D, N_{c}^{ \pm}, N_{s}^{ \pm}\right)-E_{0} \\
&= \frac{2 \pi}{L}\left[v_{c}\left(N_{c}^{+}+N_{c}^{-}\right)+v_{s}\left(N_{s}^{+}+N_{s}^{-}\right)\right] \\
&+\frac{2 \pi}{L}\left[\frac{1}{4} \Delta N^{T}\left(Z^{-1}\right)^{T} V Z^{-1} \Delta N+D^{T} Z V Z^{T} D\right] \\
&+O\left(\frac{1}{L}\right) \\
& P\left(\Delta N, D, N_{c}^{ \pm}, N_{s}^{ \pm}\right)-P_{0} \\
& \frac{2 \pi}{L}\left[v_{c}\left(N_{c}^{+}-N_{c}^{-}\right)+v_{s}\left(N_{s}^{+}-N_{s}^{-}\right)\right] \\
&+\frac{2 \pi}{L}\left[\Delta N^{T} D\right]+2 D_{c} k_{F \uparrow}+2\left(D_{c}+D_{s}\right) k_{F \downarrow}
\end{aligned}
$$

$k_{F \uparrow}=\frac{2 \pi}{L} N_{\uparrow}$ and $k_{F \downarrow}=\frac{2 \pi}{L} N_{\downarrow}$ are the Fermi momentum for particles of spin up and spin down. The energy and the momentum are those of a state with the (integer) quantum numbers $\left(\Delta N, D, N_{c}^{ \pm}, N_{s}^{ \pm}\right)$; there are two modes indexed by $c$ and $s$ : these two modes do not in general correspond to charge and spin. $Z$ is a 2 by 2 matrix:

$$
Z=\left(\begin{array}{ll}
Z_{c c} & Z_{c s} \\
Z_{s c} & Z_{s s}
\end{array}\right)
$$

and $\Delta N$ and $D$ are two-vectors $\Delta N=\left(N_{c}=N_{\uparrow}+\right.$ $\left.N_{\downarrow}, N_{s}=N_{\downarrow}\right)$ and $D=\left(D_{c}, D_{s}\right) 59$ In these expressions $N_{c / s}^{+-}$are integers: they are simply the modulus of phonon momenta in units of $2 \pi / L$ for the two modes $c$ and $s$; the index \pm refers to the sign of the momentum. The phonon velocities for the two modes are $\left(v_{c}, v_{s}\right)$.

The spectrum of the general gaussian model $H\left[u_{\tau}, \widehat{g}\right]$ is:

$$
\begin{aligned}
& E\left(Q_{\sigma}, J_{\sigma}, N_{\tau}^{ \pm}\right) \\
= & \frac{2 \pi}{L}\left[v_{\tau=1}\left(N_{\tau=1}^{+}+N_{\tau=1}^{-}\right)+v_{\tau=2}\left(N_{\tau=2}^{+}+N_{\tau=2}^{-}\right)\right] \\
& +\frac{\pi}{2 L} \sum_{\tau=1,2} v_{\tau}\left(\frac{Q_{\tau}^{2}}{K_{\tau}}+K_{\tau} J_{\tau}^{2}\right), \\
& P\left(Q_{\sigma}, J_{\sigma}, N_{\tau}^{ \pm}\right) \\
= & \frac{2 \pi}{L}\left[\left(N_{\tau=1}^{+}-N_{\tau=1}^{-}\right)+\left(N_{\tau=2}^{+}-N_{\tau=2}^{-}\right)\right]
\end{aligned}
$$




$$
+\sum_{\sigma=\uparrow \downarrow} \frac{\pi Q_{\sigma}}{L} J_{\sigma}+k_{F \sigma} J_{\sigma}
$$

$N_{\tau=1}^{ \pm}, N_{\tau=2}^{ \pm}$are again the moduli of phonon momenta. The charges and currents $\left(Q_{\tau}, J_{\tau}\right)$ are related to $\left(Q_{\sigma}, J_{\sigma}\right)$ by $Q_{\tau}=P_{\sigma \tau} Q_{\sigma}$ and $J_{\tau}=P_{\sigma \tau} J_{\sigma}$. We can now identify the parameters of both theories:

$$
\begin{aligned}
J_{\uparrow} & =2 D_{c}, J_{\downarrow}=2\left(D_{c}+D_{s}\right), \\
Q_{\uparrow} & =N_{\uparrow}, Q_{\downarrow}=N_{\downarrow}, N_{\tau}^{ \pm}=N_{c / s}^{ \pm}, \\
v_{\tau} & =v_{c / s} .
\end{aligned}
$$

The zero modes can be identified term by term; it is sufficient to consider the current terms to uniquely determine the charge matrix. The charge zero modes yield extra relations which lead to the very same expression for $\widehat{g}$. Indeed expanding the squares gives:

$$
\begin{aligned}
K_{1} P_{\uparrow 1}^{2} & =\left(Z_{c c}-Z_{s c}\right)^{2}, \\
K_{1} P_{\downarrow 1}^{2} & =\left(Z_{s c}\right)^{2}, \\
K_{1} P_{\uparrow 1} P_{\downarrow 1} & =\left(Z_{c c}-Z_{s c}\right) Z_{s c}, \\
K_{2} P_{\uparrow 2}^{2} & =\left(Z_{c s}-Z_{s s}\right)^{2}, \\
K_{2} P_{\downarrow 2}^{2} & =\left(Z_{s s}\right)^{2}, \\
K_{2} P_{\uparrow 2} P_{\downarrow 2} & =\left(Z_{c s}-Z_{s s}\right) Z_{s s} .
\end{aligned}
$$

Since:

$$
{\widehat{g^{-1}}}_{\sigma \sigma^{\prime}}=\sum_{\tau} K_{\tau} P_{\sigma \tau} P_{\sigma^{\prime} \tau}
$$

it follows that the inverse of the charge matrix is:

${\widehat{g^{-1}}}_{\uparrow \uparrow}=\left(Z_{c c}-Z_{s c}\right)^{2}+\left(Z_{c s}-Z_{s s}\right)^{2}$

$\widehat{g^{-1}} \downarrow \downarrow=\left(Z_{s c}\right)^{2}+\left(Z_{s s}\right)^{2}$

${\widehat{g^{-1}}}_{\uparrow \downarrow}={\widehat{g^{-1}}}_{\downarrow \uparrow}=\left(Z_{c c}-Z_{s c}\right) Z_{s c}+\left(Z_{c s}-Z_{s s}\right) Z_{s s}$

We define the matrix $\widetilde{Z}$ obtained from the dressed charge matrix $Z$ by subtracting the second line from the first:

$$
\widetilde{Z}=\left(\begin{array}{ll}
Z_{c c}-Z_{s c} & Z_{c s}-Z_{s s} \\
Z_{s c} & Z_{s s}
\end{array}\right)
$$

Then:

$$
\widehat{g}^{-1}=\widetilde{Z} \widetilde{Z}^{T}
$$

This is the most important result of the present section: the low-energy properties of the Hubbard model are expressed in terms of quantities which can be computed from the microscopic parameters and spin-charge separation simply follows from the $Z_{2}$ symmetry of our charge matrix. In the framework of the dressed charge matrix $Z$ approach, the second feature is not easily decoded from the structure of $Z$ which is then triangular with some relations between its matrix elements whose physical interpretation is quite unclear.60 We can use the results of the previous sections on the elementary excitations and those on the various properties of the charge matrix hamiltonian such as the Drude peak, the susceptibility, the anomalous exponents. In particular the new modes replacing the spin and charge modes are simply the eigenvectors of the charge matrix.

The charge matrix is obtained by inversion:

$$
\begin{aligned}
& \widehat{g}=\frac{1}{(\operatorname{det} Z)^{2}}
\end{aligned}
$$

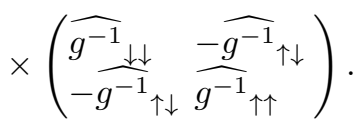

Term by term identification of the charge zero modes $Q_{\sigma}$ would lead to exactly the same expression for $\widehat{g}$; indeed:

$$
\begin{aligned}
\frac{1}{K_{1}} P_{\uparrow 1}^{2} & =\frac{\left(Z_{s s}\right)^{2}}{(\operatorname{det} Z)^{2}}, \\
\frac{1}{K_{1}} P_{\downarrow 1}^{2} & =\frac{\left(Z_{c s}-Z_{s s}\right)^{2}}{(\operatorname{det} Z)^{2}}, \\
\frac{1}{K_{1}} P_{\uparrow 1} P_{\downarrow 1} & =\frac{-\left(Z_{c s}-Z_{s s}\right) Z_{s s}}{(\operatorname{det} Z)^{2}}, \\
\frac{1}{K_{2}} P_{\uparrow 2}^{2} & =\frac{\left(Z_{s c}\right)^{2}}{(\operatorname{det} Z)^{2}}, \\
\frac{1}{K_{2}} P_{\downarrow 2}^{2} & =\frac{\left(Z_{c c}-Z_{s c}\right)^{2}}{(\operatorname{det} Z)^{2}}, \\
\frac{1}{K_{2}} P_{\uparrow 2} P_{\downarrow 2} & =\frac{-\left(Z_{c c}-Z_{s c}\right) Z_{s c}}{(\operatorname{det} Z)^{2}},
\end{aligned}
$$

and since $\widehat{g}_{\sigma \sigma^{\prime}}=\sum_{\tau} K_{\tau}^{-1} P_{\sigma \tau} P_{\sigma^{\prime} \tau}$ one recovers equ.(3.105). As it should be, one can check that the anomalous exponents predicted for $H\left[u_{\tau}, \widehat{g}\right]$ agree then completely with Frahm and Korepin's results.

Let us illustrate these results in two situations, one with spin-charge separation, the other without. From these, we can exhibit the criterion for spin-charge separation within the matrix formalism.

In the presence of spin-charge separation, Frahm and Korepin find that the dressed charge matrix $Z$ is $Z=$ $\left(\begin{array}{ll}Z_{c c}=\xi & Z_{c s}=0 \\ Z_{s c}=\xi / 2 & Z_{s s}=1 / \sqrt{2}\end{array}\right)$. This implies that $\widehat{g}$ and its inverse $\widehat{g}^{-1}$ are:

$$
\begin{aligned}
\widehat{g}^{-1} & =\left(\begin{array}{c}
\frac{\xi^{2}}{4}+\frac{1}{2} \frac{\xi^{2}}{4}-\frac{1}{2} \\
\frac{\xi^{2}}{4}-\frac{1}{2} \frac{\xi^{2}}{4}+\frac{1}{2}
\end{array}\right) \\
\widehat{g} & =\left(\begin{array}{cc}
\frac{1}{\xi^{2}}+\frac{1}{2} & \frac{1}{\xi^{2}}-\frac{1}{2} \\
\frac{1}{\xi^{2}}-\frac{1}{2} & \frac{1}{\xi^{2}}+\frac{1}{2}
\end{array}\right)
\end{aligned}
$$

The charge matrix explicitly exhibits spin-charge separation and takes the form characteristic of $S U(2)$ symmetry. The eigenvalues of $\widehat{g}^{-1}$ are $K_{c}=\frac{\xi^{2}}{2}$ and $K_{s}=1$. 
The $Z$ matrix can also be explicitly computed in the limit of infinite repulsion with a magnetic field close to the critical field $h_{c}$ for which all the spins are polarized (i.e. close to the ferromagnetic phase). In terms of the parameter

$$
\delta=\sqrt{\frac{h_{c}-h}{h_{c}}}
$$

the dressed charge matrix is:

$$
Z=\left(\begin{array}{ll}
1 & 0 \\
\frac{2}{\pi} \delta & 1-\frac{1}{\pi} \delta
\end{array}\right)
$$

which implies that the (inverse of the) charge matrix $\widehat{g}$ is:

$$
\begin{gathered}
\widehat{g}^{-1}= \\
\left(\begin{array}{ll}
\left(1-\frac{1}{\pi} \delta\right)^{2}+\left(1-\frac{2}{\pi} \delta\right)^{2} & \left(1-\frac{2}{\pi} \delta\right)\left(\frac{2}{\pi} \delta\right)-\left(1-\frac{1}{\pi} \delta\right)^{2} \\
\left(1-\frac{2}{\pi} \delta\right)\left(\frac{2}{\pi} \delta\right)-\left(1-\frac{1}{\pi} \delta\right)^{2} & \left(1-\frac{1}{\pi} \delta\right)^{2}+\left(\frac{2}{\pi} \delta\right)^{2}
\end{array}\right) .
\end{gathered}
$$

This expression shows explicitly the breakdown of spincharge separation (except for $\delta=\pi / 4$ ).

\section{CONCLUSIONS AND PERSPECTIVES.}

The main goal of our paper was to establish and describe fractional excitations for the Luttinger liquid within the bosonization scheme. bf Bethe Ansatz gives exact eigenstates and shows the existence of some fractional excitations: however their description is quite complex in that framework and it is unclear how to generate systematically a complete set of excitations. In the lowenergy limit, the Luttinger liquid approach allows a very precise characterization of the fractional states already known from exact solutions but what's more allows us to discover novel fractional excitations which may carry irrational charges (the 1D Laughlin quasiparticle, the hybrid state). In section II the low-energy spectrum of Luttinger liquids can be reinterpreted in terms of fractional states: for instance, the particle-hole continuum consists of a Laughlin quasiparticles-quasihole continuum. The quasiparticle perspective clarifies many properties of the LL: the renormalization of the current operator is a direct consequence of fractionalization; for spin chains, we present the correct description of the spinon excitation in the generic case of a violation of $S U(2)$ invariance. We also show that the $S_{z}=0$ continuum of spin chains involves the analogs of Laughlin quasiparticles. In section III we describe fractional excitations such as the holon or the spinon for the Luttinger liquid with spin; we also present a generalization of the gaussian theory valid for Luttinger liquids without spin-charge separation and display in that situation the new fractional states replacing the holon and spinon (see III g).
An important test, of course, would be to observe experimentally (or numerically) all these fractional states. Although the existence of the holon and the spinon was ascertained theoretically quite a long time ago no experiment has yet allowed their detection: in fact, the property of spin-charge separation itself is not yet established experimentally. The observation of two of the fractional states discussed in this paper would be particularly important: the LL Laughlin quasiparticle and the hybrid state. Indeed they may assume irrational charges. The precise spectroscopy of fractional excitations we have done in this paper allows us to determine which processes are involved in their creation: for the Laughlin states, current probes are needed, while the hybrid particle is created by addition of an odd number of electrons. For Laughlin quasiparticles shot noise is likely an adequate probe: the shot noise coefficient for Luttinger liquids can be computed exactly and is predicted to be equal to $K$; 63 in the two-dimensional electron gas at filling $\nu=1 / 3$ this yields a charge $1 / 32$. The latter situation involves Wen's chiral Luttinger liquid. The identification of the shot noise coefficient with the charge of a carrier has been debated because the coefficient one measures might actually be the conductance rather than a quasiparticle charge (at $\nu=1 / 3$ the conductance also assumes the value $1 / 3$ ). For the non-chiral Luttinger liquid our spectroscopy of fractional states allows to resolve that ambiguity: the shot noise coefficient is indeed identical to the conductance $K$ of the LL but the (backscattering) current-current correlation function measured in shot noise involves charge $K$ excitations, because charge $K$ LL Laughlin quasiparticles are precisely generated by current excitations. These might thus be detected in any physical realization of the Luttinger liquid: quantum wires, or possibly nanotubes. An intriguing possibility is also suggested by recent experiments of tunneling at the edge of a two-dimensional gas in a magnetic field 64. $I-V$ characteristics measured at the edge showed very surprising non-Fermi liquid behaviour compatible with a chiral LL with unquantized LL parameter $K$; the $I-V$ curves evolve smoothly when one varies the filling fraction and do not show a plateau structure. There seems to be a continuum of Luttinger liquids living at the edge: this caused quite a stir because the chiral LL theory can presumably be derived only for incompressible filling fractions. These puzzling results are sofar unexplained, but if an interpretation in terms of a single-boson mode chiral LL with unquantized parameter $K$ can be in some manner justified, according to the results given in the present paper this would imply that there exists charge $K$ Laughlin quasiparticles in that experimental setting: such a chiral LL is identical to the chiral half of the non-chiral gaussian hamiltonian considered throughout our paper.

The authors wish to acknowledge the late Heinz Schulz for insightful remarks on the results of this paper. We also thank Vincent Pasquier for a discussion on the Calogero-Sutherland model. Bernard Jancovici gave us information on one dimensional plasmas for which we are 
grateful.

\section{APPENDIX A: DISPERSION OF THE FRACTIONAL STATES.}

We show here that $V_{Q_{ \pm}}^{ \pm}(q) \mid \Psi_{0}>$ (where $\mid \Psi_{0}>$ is the interacting ground state) is an exact eigenstate of the chiral hamiltonian $H_{ \pm}$with energy:

$$
E\left(Q_{ \pm}, \overline{q_{n}}\right)=\left[u\left|\overline{q_{n}}\right|+\frac{\pi u}{2 L} \frac{Q_{ \pm}^{2}}{K}\right] .
$$

Let us rewrite the state considered $V_{Q_{ \pm}}^{ \pm}\left(q_{n}\right) \mid \Psi_{0}>$ in terms of zero modes and phonon operators (we use equ. (1.16), 1.17), (1.26) and (1.27)):

$$
\begin{gathered}
V_{Q_{ \pm}}^{ \pm}(x) \mid \Psi_{0}>=\exp i Q_{ \pm}\left[\mp \frac{2 \pi}{K} \widehat{Q}_{ \pm} x-\sqrt{\pi}\left(\Theta_{0} \mp \frac{\Phi_{0}}{K}\right)\right] \\
\times \exp -i \sqrt{\pi} Q_{ \pm} \sum_{n \neq 0} \Theta_{ \pm, n} \exp i \frac{2 \pi n}{L} x \mid \Psi_{0}>
\end{gathered}
$$

Taking into account the fact that the operators $b_{q}$ annihilate the ground state, it follows that:

$$
\begin{aligned}
& V_{Q_{+}}^{+}(x) \mid \Psi_{0}>=\exp i Q_{+}\left[-\frac{2 \pi}{K} \widehat{Q}_{+} x-\sqrt{\pi}\left(\Theta_{0}-\frac{\Phi_{0}}{K}\right)\right] \\
& \left.\times \exp -i \sqrt{\pi} Q_{+} \sum_{n>0} \sqrt{\frac{L}{K \pi|n|}} b_{n}^{+} \exp i \frac{2 \pi n}{L} x \mid \Psi_{0}>, \quad \text { (A3 }\right)
\end{aligned}
$$

with a similar expression for $V_{Q_{-}}^{-}(x) \mid \Psi_{0}>$ (the sum is then over negative momentum phonons). Going back to reciprocal space:

$$
\begin{aligned}
& V_{Q_{ \pm}}^{ \pm}\left(q_{n}\right) \mid \Psi_{0}>=\frac{1}{\sqrt{L}} \int_{0}^{L} d x \exp -i \frac{2 \pi}{L} n x \\
& \times \exp i Q_{+}\left[-\sqrt{\pi}\left(\Theta_{0} \mp \frac{\Phi_{0}}{K}\right)\right] \\
& \times \exp -i \sqrt{\pi} Q_{+} \sum_{ \pm p>0} \sqrt{\frac{L}{K \pi|p|}} b_{p}^{+} \exp i \frac{2 \pi p}{L} x \mid \Psi_{0}>,
\end{aligned}
$$

which shows that $V_{Q_{ \pm}}^{ \pm}\left(q_{n}\right) \mid \Psi_{0}>$ only spans chiral phonons with momentum $\pm n>0$; in other words this state is obtained by the action of the zero mode of the chiral field plus the creation of phonons with momenta of the same sign. When one expands the phonon exponential, the integral over position will select configuration of phonons with identical total momentum $\overline{q_{n}}=\frac{2 \pi n}{L}$. All these configurations consist of phonons of identical chirality and total momentum which means that they are eigenstates with the same eigenvalue of the appropriate chiral hamiltonian $\left(H_{+}\right.$or $\left.H_{-}\right)$. This is enough to prove that $V_{Q_{ \pm}}^{ \pm}\left(q_{n}\right) \mid \Psi_{0}>$ is an exact eigenstate of $H_{ \pm}$:

$$
\begin{aligned}
H_{ \pm} V_{Q_{ \pm}}^{ \pm}\left(q_{n}\right) \mid \Psi_{0} & >=\left[\sum_{q>0} u|q| a_{q}^{+} a_{q}+\frac{\pi u}{2 L} \frac{\widehat{Q}_{ \pm}^{2}}{K}\right] \\
\times V_{Q_{ \pm}}^{ \pm}\left(q_{n}\right) \mid \Psi_{0} & > \\
& =\left[u\left|\frac{2 \pi n}{L}\right|+\frac{\pi u}{2 L} \frac{Q_{ \pm}^{2}}{K}\right] V_{Q_{ \pm}}^{ \pm}\left(q_{n}\right) \mid \Psi_{0}>
\end{aligned}
$$

where $\overline{q_{n}}=\frac{2 \pi n}{L}$ is the momentum due to phonons.

${ }^{1}$ R. B. Laughlin, Phys. Rev. Lett. 50, 1395 (1983)

${ }^{2}$ L. Saminadayar, D. C. Glattli, Y. Jin, B. Etienne, Phys. Rev. Lett. 79 2526(1997); R. de Picciotto, M. Reznikov, M. Heiblum, V. Umansky, G. Bunin, D. Mahalu, Nature 389, 162 (1997)

${ }^{3}$ J. Hubbard, Proc. Roy. Soc. A 240, 539 (1957); 243, 336 (1958); 276, 238 (1963).

${ }^{4}$ H. A. Bethe, Z. Phys. 71, 205 (1931).

${ }^{5}$ E. H. Lieb and F. Y. Wu, Phys. Rev. Lett. 20, 1445 (1968).

${ }^{6}$ L. D. Fadeev, L. A. Takhtajan, Phys. Lett. A 85, 375 (1981)

7 J. D. Johnson, S. Krinsky, B. M. McCoy, Phys.Rev. A 8, 2526 (1972)

${ }^{8}$ F. D. M. Haldane, Phys. Rev. Lett. 45, 1358 (1980); ibid, J. Phys. C 14, 2585 (1981)

${ }^{9}$ L. D. Landau, Sov. Phys. JETP 3, 920 (1957); 5, 101 (1957); 8, 70 (1959).

10 J. M. Luttinger, J. Math. Phys. 4, 1154 (1963).

${ }^{11}$ S. Tomonaga, Prog. Theor. Phys. 5, 544 (1950).

12 D. C. Mattis and E. H. Lieb, J. Math. Phys. 6, 304 (1963).

${ }^{13}$ C. Bourbonnais, L.G. Caron, Int. J. Mod.Phys.B 5, 1033 (1991); R.Shankar, Rev. Mod. Phys. 66, 129 (1994)

${ }^{14}$ We call these excitations describing collective density fluctuations interchangeably phonons, or plasmons.

${ }^{15} c$ is a real number known as the conformal anomaly.

${ }^{16}$ A. A. Belavin, A. M. Polyakov, and A. B. Zamolodchikov, Nucl. Phys. B 241, 333 (1984); C. Itzykson and J.-M. Drouffe, Statistical Field Theory, Cambridge Universtity Press, Cambridge, 1989, vol. 2; P. Ginsparg, in Fields, Strings and Critical Phenomena, Les Houches Summer School 1988, Eds. E.Brezin, J.Zinn-Justin (NorthHolland, Amsterdam, 1990).

${ }^{17}$ H. W. J. Blöte, J. L. Cardy, and M. P. Nightingale, Phys. Rev. Lett. 56, 742 (1986)

18 N. Kawakami, S. K. Yang, Phys.Rev.Lett. 67, 2493 (1991)

19 P. Bouwknegt, A. Ludwig, K. Scoutens, Phys. Lett. B 338, 448 (1994); K. Schoutens, Phys. Rev. Lett. 79, 2608 (1997)

${ }^{20}$ O. Babelon, H. de Vega, and C.M Viallet, Nucl. Phys. B 220, 13 (1983); K. Sogo, Phys. Lett. A 104, 51 (1984).

${ }^{21}$ F. D. M.Haldane, Phys. Rev. Lett. 60, 635 (1988); B. S. Shastry, Phys. Rev. Lett. 60, 639 (1988) 
${ }^{22}$ F. D. M. Haldane, in Proceedings of the 16th Taniguchi Symposium on Condensed Matter Physics, Kagoshima, Japan, 1993, Eds A.Ojiki, N.Kawakami (Springer, BerlinHeidelberg-New-York, 1994)

${ }^{23}$ We omitted a phase factor corresponding to a momentum $\pi$ boost. It can be ignored after a bipartite transformation.

${ }^{24}$ F. Woynarowich, J. Phys. A 15, 2985 (1982)

${ }^{25}$ H. Frahm, V. E. Korepin, Phys. Rev. B 42, 10553 (1990); ibid, Phys. Rev. B 43, 5653 (1991)

${ }^{26}$ F. Calogero, J. Math. Phys. 10, 2191(1969); B. Sutherland, Phys. Rev. A 4, 2019 (1971)

${ }^{27}$ M. R. Zirnbauer, F. D. M. Haldane, Phys. Rev. B 52, 8729 (1995);

${ }^{28}$ X. G. Wen, Phys. Rev. B 41, 12838 (1990); X. G. Wen, Adv. Phys. 44, 405 (1995)

29 Z. N. C. Ha, Phys. Rev. Lett 73, 1574 (1994)

${ }^{30}$ M. P. A. Fisher, L. I. Glazman, in Mesoscopic electron transport, ed. by L. Kovenhoven, G. Schoen, L. Sohn (Kluwer, Dordrecht).

${ }^{31}$ R. Heidenreich, R. Seiler, and A. Uhlenbrock, J. Stat. Phys. 22, 27 (1980).

32 K-V Pham, M. Gabay, P. Lederer, Eur. Phys. Journ. B 9, 573 (1999)

${ }^{33}$ G. V. Chester, L. Reatto, Phys. Lett. 22, 3 (1966); R. P. Feynman, Statistical Physics, (Addison-Wesley, New-York, 1972)

${ }^{34}$ F. D. M. Haldane, Phys. Rev. Lett. 47, 1840 (1981)

35 The zero mode basis (phonons and $U_{ \pm}$operators) forms a complete family of states: this was proved by Haldane in his computation of the grand canfnical partition function of the Tomonaga-Luttinger model 8 . Therefore $V_{Q, J}$ operators span the Fock space since $V_{Q, J}$ operators are products of Haldane's $U_{ \pm}$operators and of an exponential of phonons. Since the fractional states $V_{Q_{ \pm}}^{ \pm}$come from the decay of the $V_{Q, J}$ operators, they, along with the phonons span the Fock space.

${ }^{36}$ This is just the quantization of the superfluid phase: the boson operator is $\Psi_{B} \propto \exp i \sqrt{\pi} \Theta$ and therefore $\sqrt{\pi}(\Theta(L)-\Theta(0))$ must be an integer multiple of $\pi$. The total current around the ring is defined as the integral $\int_{0}^{L} d x j_{R}(x)=\widehat{J}_{R}$; therefore $\widehat{J}_{R}=\frac{u K}{\sqrt{\pi}}(\Theta(L)-\Theta(0))$ and we may then write $\widehat{J}_{R}=u K J$ where $J$ is an integer.

37 The previous equation assumes that both $Q$ and $n$ are positive; if that is not so, it suffices to take their absolute value and replace the operators by their conjugate: for instance $Q \longrightarrow|Q|$ and $\left[W_{1 / 2}^{ \pm}(x)\right] \longrightarrow\left[W_{-1 / 2}^{ \pm}(x)\right]$.

${ }^{38}$ D. Bohm, D. Pines, Phys. Rev. 85, 338 (1952)

${ }^{39}$ Recall that bosonization formulae for the electron operators are obtained by making a Jordan-Wigner transformation on the boson operators; likewise the fermionic $(Q, J)$ selection rules $(Q+J$ even) derive directly from the change in statistics: a $(Q, J)$ excitation of the bosonic LL becomes a $\left(Q, J^{\prime}=J+Q\right)$ states due to the Jordan-Wigner transformation which attaches one flux-tube to each particle $(Q$ tubes to $Q$ particles, which increases $J$ by $Q$ units). Since $J$ is even for the bosonic LL, $Q+J^{\prime}$ is even for the fermionic LL.

${ }^{40}$ S. C. Zhang, H. Hansson, S. Kivelson, Phys. Rev. Lett. 62, 82 (1989); S. C. Zhang, Int. J. Mod. Phys. B 6, 25 (1992)
${ }^{41}$ Here and in the remaining of the paper for convenience we will consider the operators in real space and the wavefunctions will be localized: the exact eigenfunctions are of course recovered by taking the Fourier transform of the localized (Wannier) states.

42 B. Horowitz, Phys. Rev. Lett 48, 1416 (1982)

43 A. Luther, I. Peschel, Phys. Rev. B 12, 3908 (1975)

44 The Jordan-Wigner transformation which maps spins onto fermions might lead one to think that the spectrum of the spin chain is identical to that of the Jordan-Wigner fermions. This is correct only for the spectrum of an even number of fermions added in (this corresponds to spin transitions $\Delta S_{z}=2 p$ where $p$ is an integer): imposing periodic $(\mathrm{PBC})$ or anti-periodic (ABC) boundary conditions forces the number of fermions to keep a given parity (even for $\mathrm{PBC}$ and odd for $\mathrm{ABC}$ ), thus the mapping of spins onto fermions is not valid when one adds an odd number of fermions to the ground state. The spin flip operator is not a Jordan-Wigner fermion since one also creates a flux tube (the Jordan-Wigner string). This has led to some confusion in the literature. The $\Delta S_{z}=0$ continuum is correctly described by the excitations of the Jordan-Wigner fermion system since this corresponds to the addition of an even number of fermions. For the $X X$ spin chain this means that the $\Delta S_{z}=0$ continuum is due to spin one states which are Jordan-Wigner fermions. But if the mapping were also valid for $\Delta S_{z}=1$ excitations, this would mean that the continuum is also due to spin one states. This is not correct because as we know this latter continuum is due to pairs of spinons.

${ }^{45}$ By adiabaticity, we mean a finite quasiparticles weight, as for the Landau quasiparticles which is an adiabatic deformation of the screened free electron.

46 J. C. Talstra, S. P. Strong, Phys. Rev. B 56, 6094 (1997)

${ }^{47}$ I. Safi, PhD Thesis, Université de Paris, Orsay, unpublished; ibid, Annales de Physique 22, 463 (1997)

${ }^{48}$ M. Stone, M. P A. Fisher, Int. Journ. Mod. Phys. B 8, 2539 (1994)

${ }^{49}$ K.-V. Pham, PhD thesis, Université de Paris, Orsay, unpublished.

${ }^{50}$ B. I. Halperin, Helv. Phys. Acta 56, 75 (1983)

${ }^{51}$ The $\widehat{g}$ matrix is distinguished from the $K$ matrix to avoid a possible confusion because its eigenvalues are $1 / K_{c}$ and $1 / K_{s}$.

${ }^{52}$ It would be more correct to call this operator an anti-holon since its electrical charge is $Q_{e}=(-e) Q=-e$; but apart from the sign of the charge all fractional particles considered in this paper and their particle-hole conjugates have the same properties. So we call this state a holon.

${ }^{53}$ A naive decoupling of the electron operator would lead to:

$$
\begin{aligned}
\Psi_{\sigma}= & \exp -i \sqrt{\pi}\left(\Theta_{\sigma}-\Phi_{\sigma}\right) \\
= & \exp -i \sqrt{\pi / 2}\left(\Theta_{c}-\Phi_{c}\right) \\
& \exp -i \sqrt{\pi / 2}\left(\Theta_{s}-\Phi_{s}\right)
\end{aligned}
$$

The first term is sometimes identified as the holon and it has a semionic statistics. The second term is likewise supposed to be the spinon. These identifications are in general incorrect because these states are unstable because of chiral separation (see the anisotropic spin chain). In the spe- 
cial case of $S U(2)$ symmetry, the above expression for the spinon is accidentally correct.

${ }^{54}$ C. S. Hellberg and E. J. Mele, Phys. Rev. Lett. 67, 2080 (1991).

${ }^{55}$ M. Ogata, H. Shiba, Phys. Rev. B 41, 2326 (1990)

${ }^{56}$ F. Woynarovich, J. Phys. A 22, 2115 (1990)

${ }^{57}$ K. Penc and J. Sólyom, Phys. Rev. B 47, 6273 (1993)

58 The ambiguity which is rather technical is the following: there are four kinds of anomalous exponents (two chiralities and two modes). For a given excitation in a given mode, the sum of anomalous exponents for the two chiralities is fixed but their difference is not. The dimensions are therefore only known up to a constant; to fix their values, Frahm and Korepin make the unproven assumption that they are perfect squares if the excitation corresponds to a primary field.

${ }^{59}$ Note that in Frahm and Korepin' notations $N_{s}=N_{\downarrow}$ is not the spin density.

60 The charge matrix and its inverse have the same properties, $Z_{2}$ symmetry or $S U(2)$ symmetry in the relevant cases.

${ }^{61}$ P. W. Anderson, Physics Today, 50, ${ }^{\circ}:$ 10, pp 42-47 (1997)

${ }^{62}$ W. P. Su, J. R. Schrieffer, A. J. Heeger, Phys. Rev. Lett. 42, 1698 (1979)

${ }^{63}$ P. Fendley, A. Ludwig, H. Saleur, Phys. Rev. Lett. 74, 3005 (1994)

${ }^{64}$ M. Grayson et al, Phys.Rev.Lett. 80, 1062 (1998) 
FIG. 1. Spectrum of the gaussian model with a bosonic Fock space in the charge $Q$ sector. The energy at zero momentum is $\Delta(Q)=\pi u Q^{2} /(2 L)$ as a function of $Q$ the number of particles added to the system. The spectrum for fermionic Fock spaces is identical if $Q$ is an even integer. The continuum is enclosed within the straight lines which are supported by the parabolic enveloppe $\pi u\left(k / k_{F}\right)^{2} /(2 L)$. 
FIG. 2. Spectrum of the gaussian model for fermionic Fock spaces in the case of charge sectors for which $Q$ is an odd integer. Notice that the energy has now local minima for momenta $k= \pm 1, \pm 3, \pm 5, \ldots$ (in units of $k_{F}$ ) instead of $k=0, \pm 2, \pm 4, \ldots$ 
FIG. 3. Spectrum of the $X X Z$ spin chain for a transverse exchange $J$ and anisotropy $\Delta$; the continuum is enclosed within the curves $E(k)=(\pi \alpha / 2)|\sin (k)|$ and $E(k)=\pi \alpha \sin (k / 2)$. The parameter $\alpha$ is related to the anisotropy $\Delta=\cos \theta$ by $\alpha=\sin \theta / \theta$. The linearized spectrum found by bosonization is also shown. 